ESAIM: COCV 18 (2012) 1073-1096

DOI: $10.1051 / \mathrm{cocv} / 2011204$
ESAIM: Control, Optimisation and Calculus of Variations

www.esaim-cocv.org

\title{
MAXIMUM PRINCIPLE FOR OPTIMAL CONTROL OF FULLY COUPLED FORWARD-BACKWARD STOCHASTIC DIFFERENTIAL DELAYED EQUATIONS *
}

\author{
JiANHUI HUANG ${ }^{1}$ AND JingtaO SHI ${ }^{2}$
}

\begin{abstract}
This paper deals with the optimal control problem in which the controlled system is described by a fully coupled anticipated forward-backward stochastic differential delayed equation. The maximum principle for this problem is obtained under the assumption that the diffusion coefficient does not contain the control variables and the control domain is not necessarily convex. Both the necessary and sufficient conditions of optimality are proved. As illustrating examples, two kinds of linear quadratic control problems are discussed and both optimal controls are derived explicitly.
\end{abstract}

Mathematics Subject Classification. 93E20, 60H10, 34K50.

Received August 12, 2010. Revised August 13, 2011.

Published online 16 January 2012.

\section{INTRODUCTION AND PROBLEM FORMULATION}

Throughout this paper, $\mathbf{R}^{n}$ denotes the $n$-dimensional Euclidean space. $\langle\cdot, \cdot\rangle$ and $|\cdot|$ denote the scalar product and norm in the Euclidean space, respectively. T appearing in the superscripts denotes the transpose of a matrix. $C>0$ denotes a constant which can be changed line by line.

Let $(\Omega, \mathcal{F}, \mathbf{P})$ be a complete filtered probability space equipped with a natural filtration $\mathcal{F}_{t}:=\sigma\{W(s) ; 0 \leq$ $s \leq t\}$, where $W(\cdot)$ is a $d$-dimensional standard Brownian motion. Let $T>0$ be a fixed time horizon. $\mathbb{E}$ denotes the expectation under $\mathbf{P}$ and $\mathbb{E}^{\mathcal{F}_{t}}[\cdot]:=\mathbb{E}\left[\cdot \mid \mathcal{F}_{t}\right]$ denotes the conditional expectation. $L^{2}\left(\Omega, \mathcal{F}_{T} ; \mathbf{R}^{n}\right)$ denotes the space of all $\mathbf{R}^{n}$-valued $\mathcal{F}_{T}$-measurable random variables $\xi$ satisfying $\mathbb{E}\left[|\xi|^{2}\right]<\infty, L_{\mathcal{F}}^{2}\left([0, T] ; \mathbf{R}^{n}\right)$ denotes the

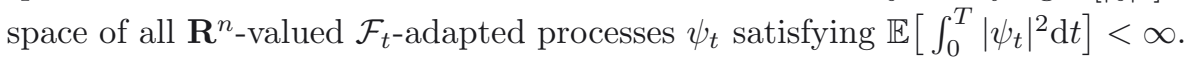

\footnotetext{
Keywords and phrases. Stochastic optimal control, maximum principle, stochastic differential delayed equation, anticipated backward differential equation, fully coupled forward-backward stochastic system, Clarke generalized gradient.

* Jianhui Huang was supported by Departmental General Research Fund (No. A-SA69) and RGC Earmarked Grants of The Hong Kong Polytechnic University (Nos. 500909, 501010). Jingtao Shi was supported by China Postdoctoral Science Foundation Funded Project (No. 20100481278), Postdoctoral Innovation Foundation Funded Project of Shandong Province (No. 201002026), National Natural Sciences Foundations of China (No. 11126209) and Shandong Province (No. ZR2011AQ012), Research Award Fund for Outstanding Young and Middle-aged Scientists of Shandong Province (No. BS2011SF010), and Independent Innovation Foundation of Shandong University (IIFSDU, No. 2010TS060).

1 Department of Applied Mathematics, The Hong Kong Polytechnic University, Hung Hom, Kowloon, Hong Kong, P.R. China. majhuang@inet.polyu.edu.hk

2 School of Mathematics, Shandong University, Jinan 250100, P.R. China. shijingtao@sdu.edu.cn
} 
Let nonempty $\mathbf{U} \subset \mathbf{R}^{k}$. Admissible control $\mathcal{U}_{a d}$ is the set of $\mathcal{F}_{t}$-adapted processes $v: \Omega \times[0, T] \rightarrow \mathbf{U}$ satisfying $\sup _{0 \leq t \leq T} \mathbb{E}|v(t)|^{4}<\infty$. For given $v(\cdot) \in \mathcal{U}_{a d}$, consider the following control system described by a fully-coupled anticipated forward-backward stochastic differential delayed equation (AFBSDDE):

$$
\left\{\begin{aligned}
\mathrm{d} x^{v}(t)= & b\left(t, x^{v}(t), y^{v}(t), z^{v}(t), x^{v}(t-\delta), v(t), v(t-\delta)\right) \mathrm{d} t \\
& \quad+\sigma\left(t, x^{v}(t), y^{v}(t), z^{v}(t), x^{v}(t-\delta)\right) \mathrm{d} W(t), \quad t \in[0, T], \\
& -\mathrm{d} y^{v}(t)=f\left(t, x^{v}(t), y^{v}(t), z^{v}(t), \mathbb{E}^{\mathcal{F}_{t}}\left[y^{v}(t+\delta)\right], v(t), v(t-\delta)\right) \mathrm{d} t-z^{v}(t) \mathrm{d} W(t), \quad t \in[0, T], \\
x^{v}(t)= & \xi(t), v(t)=\eta(t), \quad t \in[-\delta, 0], \\
y^{v}(T)= & g\left(x^{v}(T)\right), y^{v}(t)=\varphi(t), \quad t \in(T, T+\delta] .
\end{aligned}\right.
$$

Here triple $\left(x^{v}(t), y^{v}(t), z^{v}(t)\right): \Omega \times[-\delta, T] \times[0, T+\delta] \times[0, T] \rightarrow \mathbf{R}^{n} \times \mathbf{R}^{m} \times \mathbf{R}^{m \times d}$ are called state trajectory corresponding to $v(\cdot) . b:[0, T] \times \mathbf{R}^{n} \times \mathbf{R}^{m} \times \mathbf{R}^{m \times d} \times \mathbf{R}^{n} \times \mathbf{U} \times \mathbf{U} \rightarrow \mathbf{R}^{n}, \sigma:[0, T] \times \mathbf{R}^{n} \times \mathbf{R}^{m} \times \mathbf{R}^{m \times d} \times \mathbf{R}^{n} \rightarrow$ $\mathbf{R}^{n \times d}, f:[0, T] \times \mathbf{R}^{n} \times \mathbf{R}^{m} \times \mathbf{R}^{m \times d} \times \mathbf{R}^{m} \times \mathbf{U} \times \mathbf{U} \rightarrow \mathbf{R}^{m}, g: \mathbf{R}^{n} \rightarrow \mathbf{R}^{m}$ are given continuous functions. $\delta>0$ is a given finite time delay. $\xi(\cdot) \in L_{\mathcal{F}}^{2}\left([-\delta, 0] ; \mathbf{R}^{n}\right)$ is the initial path of $x^{v}(\cdot), \eta(\cdot)$ is the initial path of control $v(\cdot)$ satisfying $\sup _{-\delta<t \leq 0} \mathbb{E}|\eta(t)|^{4}<\infty$ and $\varphi(\cdot) \in L_{\mathcal{F}}^{2}\left([T, T+\delta] ; \mathbf{R}^{m}\right)$ is the terminal path of $y^{v}(\cdot)$.

We are given an $m \times n$ full-rank matrix $G$. For any $v(\cdot) \in \mathcal{U}_{a d}$, for simplicity we denote $x^{v}(t-\delta), \mathbb{E}^{\mathcal{F}_{t}}\left[y^{v}(t+\right.$ $\delta)], v(t-\delta)$ by $x^{v}(\delta), y^{v}(+\delta), v(\delta)$, respectively. And we use the following notations:

$$
\Gamma^{v}:=\left(\begin{array}{l}
x^{v} \\
y^{v} \\
z^{v}
\end{array}\right), \quad \mathcal{A}\left(t, \Gamma^{v}, x^{v}(\delta), y^{v}(+\delta), v, v(\delta)\right):=\left(\begin{array}{c}
-G^{\top} f\left(t, \Gamma^{v}(t), y^{v}(+\delta), v(t), v(\delta)\right) \\
G b\left(t, \Gamma^{v}(t), x^{v}(\delta), v(t), v(\delta)\right) \\
G \sigma\left(t, \Gamma^{v}(t), x^{v}(\delta)\right)
\end{array}\right)
$$

here $G \sigma \equiv\left(G \sigma_{1}, G \sigma_{2}, \ldots, G \sigma_{d}\right)$. Given $v(\cdot) \in \mathcal{U}_{a d}$, we assume that the following hypothesis holds.

(i) For all $\left(\Gamma^{v}, x^{v}(\delta), y^{v}(+\delta)\right), \mathcal{A}\left(t, \Gamma^{v}, x^{v}(\delta), y^{v}(+\delta), v, v(\delta)\right) \in L_{\mathcal{F}}^{2}\left([0, T] ; \mathbf{R}^{n} \times \mathbf{R}^{m} \times \mathbf{R}^{m \times d}\right)$;

(ii) For all $t \in[0, T]$, there exists a constant $C>0$, such that

$$
\begin{aligned}
& \left|\mathcal{A}\left(t, \Gamma^{v}, x^{v}(\delta), y^{v}(+\delta), v, v(\delta)\right)\right|-\left|\mathcal{A}\left(t, \bar{\Gamma}^{v}, \bar{x}^{v}(\delta), \bar{y}^{v}(+\delta), v, v(\delta)\right)\right| \\
& \leq C\left(\left|\Gamma^{v}-\bar{\Gamma}^{v}\right|+\left|x^{v}(\delta)-\bar{x}^{v}(\delta)\right|+\left|y^{v}(+\delta)-\bar{y}^{v}(+\delta)\right|\right), \forall \Gamma^{v}=\left(x^{v}, y^{v}, z^{v}\right), \bar{\Gamma}^{v}=\left(\bar{x}^{v}, \bar{y}^{v}, \bar{z}^{v}\right)
\end{aligned}
$$

(iii) $b, \sigma$ is continuously differentiable in $\left(\Gamma^{v}, x^{v}(\delta)\right)$ with uniformly bounded partial derivatives;

(iv) $f$ is continuously differentiable in $\left(\Gamma^{v}, y^{v}(+\delta)\right)$ with uniformly bounded partial derivatives;

(v) $g$ is Lipschitz continuous and continuously differentiable with uniformly bounded derivative;

(vi) For each $x \in \mathbf{R}^{n}, g(x) \in L^{2}\left(\Omega, \mathcal{F}_{T} ; \mathbf{R}^{m}\right)$.

or

$$
(\mathbf{H 2})\left\{\begin{array}{l}
\int_{0}^{T}\left\langle\mathcal{A}\left(t, \Gamma^{v}, x^{v}(\delta), y^{v}(+\delta), v, v(\delta)\right)-\mathcal{A}\left(t, \bar{\Gamma}^{v}, \bar{x}^{v}(\delta), \bar{y}^{v}(+\delta), v, v(\delta)\right), \Gamma^{v}-\bar{\Gamma}^{v}\right\rangle \mathrm{d} t \\
\quad \leq \int_{0}^{T}\left[-\beta_{1}\left|G \hat{x}^{v}\right|^{2}-\beta_{2}\left(\left|G^{\top} \hat{y}^{v}\right|^{2}+\left|G^{\top} \hat{z}^{v}\right|^{2}\right)\right] \mathrm{d} t, \\
\left\langle g\left(x^{v}\right)-g\left(\bar{x}^{v}\right), G\left(x^{v}-\bar{x}^{v}\right)\right\rangle \geq \mu_{1}\left|G \hat{x}^{v}\right|^{2},
\end{array}\right.
$$

$$
(\mathbf{H 2})^{\prime}\left\{\begin{array}{l}
\int_{0}^{T}\left\langle\mathcal{A}\left(t, \Gamma^{v}, x^{v}(\delta), y^{v}(+\delta), v, v(\delta)\right)-\mathcal{A}\left(t, \bar{\Gamma}^{v}, \bar{x}^{v}(\delta), \bar{y}^{v}(+\delta), v, v(\delta)\right), \Gamma^{v}-\bar{\Gamma}^{v}\right\rangle \mathrm{d} t \\
\geq \int_{0}^{T}\left[\beta_{1}\left|G \hat{x}^{v}\right|^{2}+\beta_{2}\left(\left|G^{\top} \hat{y}^{v}\right|^{2}+\left|G^{\top} \hat{z}^{v}\right|^{2}\right)\right] \mathrm{d} t, \\
\left\langle g\left(x^{v}\right)-g\left(\bar{x}^{v}\right), G\left(x^{v}-\bar{x}^{v}\right)\right\rangle \leq-\mu_{1}\left|G \hat{x}^{v}\right|^{2},
\end{array}\right.
$$


for all $\Gamma^{v}=\left(x^{v}, y^{v}, z^{v}\right), \bar{\Gamma}^{v}=\left(\bar{x}^{v}, \bar{y}^{v}, \bar{z}^{v}\right), \hat{x}^{v}=x^{v}-\bar{x}^{v}, \hat{y}^{v}=y^{v}-\bar{y}^{v}, \hat{z}^{v}=z^{v}-\bar{z}^{v}$. Here $\beta_{1}, \beta_{2}$ and $\mu_{1}$ are given nonnegative constants with $\beta_{1}+\beta_{2}>0, \beta_{2}+\mu_{1}>0$. Moreover we have $\beta_{1}>0, \mu_{1}>0$ (resp., $\beta_{2}>0$ ), when $m>n$ (resp., $m<n)$.

We can see that in (1.1), the forward part with initial condition is a stochastic differential delayed equation (SDDE) and the backward part with terminal condition is an anticipated backward stochastic differential equation (ABSDE). The general theory and applications of SDDEs and ABSDEs can be found in Mohammed [14] and Peng and Yang [20], respectively. In addition, one distinguished feature of equation (1.1) is that the forward SDDE and backward ABSDE are fully coupled. The existence and uniqueness of the solution of such AFBSDDE under the above $G$-monotonic assumptions has been studied recently by Chen and Wu [6] (noting that this kind $G$-monotonic assumption was initially introduced by $\mathrm{Hu}$ and Peng [11], Peng and $\mathrm{Wu}[19])$.

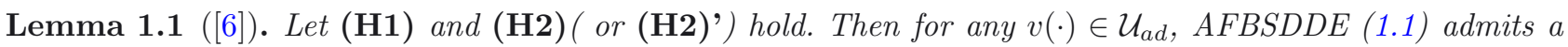
unique adapted solution $\left(x^{v}(\cdot), y^{v}(\cdot), z^{v}(\cdot)\right) \in L_{\mathcal{F}}^{2}\left([-\delta, T] ; \mathbf{R}^{n}\right) \times L_{\mathcal{F}}^{2}\left([0, T+\delta] ; \mathbf{R}^{m}\right) \times L_{\mathcal{F}}^{2}\left([0, T] ; \mathbf{R}^{m \times d}\right)$.

We introduce the following cost functional

$$
J(v(\cdot))=\mathbb{E}\left[\int_{0}^{T} l\left(t, x^{v}(t), y^{v}(t), z^{v}(t), x^{v}(t-\delta), v(t), v(t-\delta)\right) \mathrm{d} t+\Phi\left(x^{v}(T)\right)+\gamma\left(y^{v}(0)\right)\right],
$$

here $l:[0, T] \times \mathbf{R}^{n} \times \mathbf{R}^{m} \times \mathbf{R}^{m \times d} \times \mathbf{R}^{n} \times \mathbf{U} \times \mathbf{U} \rightarrow \mathbf{R}, \Phi: \mathbf{R}^{n} \rightarrow \mathbf{R}, \gamma: \mathbf{R}^{n} \rightarrow \mathbf{R}$ are given continuous functions. We need the following hypothesis.
(H3) $\left\{\begin{array}{l}\text { (i) } l \text { is continuously differentiable in } \Gamma^{v}, x^{v}(\delta) \text {, with partial derivatives being continuous } \\ \text { in }\left(\Gamma^{v}, x^{v}(\delta), v, v(\delta)\right) \text { and bounded by } C\left(1+\left|x^{v}\right|+\left|y^{v}\right|+\left|z^{v}\right|+\left|x^{v}(\delta)\right|+|v|+|v(\delta)|\right) \text {; } \\ \text { (ii) } \Phi \text { is continuously differentiable and } \Phi_{x} \text { is bounded by } C\left(1+\left|x^{v}\right|\right) \text {; }\end{array}\right.$
(ii) $\Phi$ is continuously differentiable and $\Phi_{x}$ is bounded by $C\left(1+\left|x^{v}\right|\right)$;
(iii) $\gamma$ is continuously differentiable and $\gamma_{y}$ is bounded by $C\left(1+\left|y^{v}\right|\right)$.

Our stochastic optimal control problem is to minimize the cost functional (1.2) over $v(\cdot) \in \mathcal{U}_{\text {ad }}$ subject to (1.1), i.e., to find a $u(\cdot) \in \mathcal{U}_{a d}$ satisfying

$$
J(u(\cdot))=\inf _{v(\cdot) \in \mathcal{U}_{a d}} J(v(\cdot)) .
$$

Our main target in this paper is to find some necessary condition of the stochastic optimal control $u(\cdot)$ in the form of Pontryagin's type maximum principle. We also investigate when the derived maximum principle becomes sufficient condition. To get it, we introduce the following additional assumptions.

(H4) $g(x) \equiv M_{T} x, M_{T} \in \mathbf{R}^{m \times n}, \forall x \in \mathbf{R}^{n} ; \Phi$ is convex in $x, \gamma$ is convex in $y$.

The optimal control problem for stochastic differential delayed systems has been studied by many researchers (see Kolmanovskii and Maizenberg [12], Øksendal and Sulem [15], Chen and Wu [5] and the references therein) which has the practical background. One of the main motivations is that many random phenomena have the feature of past-dependence, i.e., their behavior at time $t$ depends not only on the situation at $t$, but also on a finite part of their past history. For example, the evolution of the stock price and other stochastic dynamical systems are sometimes identified as SDDEs.

General nonlinear BSDEs were developed by Pardoux and Peng [16] and have been widely applied in optimal control, finance and partial differential equations (see Peng [17,18], El Karoui et al. [10], Yong and Zhou [27]). 
Recently, Chen and $\mathrm{Wu}[5]$ studied one kind of delayed stochastic optimal control problem. When introducing the adjoint equation, they encountered some new type of BSDEs. This new type of BSDEs was already introduced, also recently, by Peng and Yang [20] for the general nonlinear case and they called them anticipated BSDEs (ABSDEs). Moreover, they find that there exists a duality relation between SDDE and ABSDE.

Forward-backward stochastic systems where the controlled systems are described by forward-backward stochastic differential equations (FBSDEs) are widely used in mathematical economics and mathematical finance. They are encountered in stochastic recursive utility optimization problems (see Antonelli [1], El Karoui et al. [10], Wang and $\mathrm{Wu}$ [23]) and principal-agent problems (see Williams [24], Cvitanic et al. [9]). Moreover, some financial optimization problems for large investors (see Cvitanic and Ma [8], Cucoo and Cvitanic [7], Buckdahn and $\mathrm{Hu}[3]$ ) and some asset pricing problems with forward-backward differential utility (see Antonelli [1], Antonelli et al. [2]) will directly lead to fully coupled FBSDEs. So the optimal control problems for forwardbackward stochastic systems are extensively studied in the literature.

However, to our best knowledge, there are few papers studying forward-backward stochastic differential delayed systems. Recently, Chen and $\mathrm{Wu}$ [4] discussed one kind of stochastic recursive optimal control problem of the system described by FBSDE with time-varying delay. The necessary condition for the optimal control maximum principle - is derived. In this paper, we will further research on this topic and consider the optimal control problem for stochastic system described by the fully coupled AFBSDDE (1.1). Our work distinguishes itself from $[4,5]$ in the following aspects:

(i) The control system (1.1) itself is a fully coupled AFBSDDE. First of all, in [5] the state equation and adjoint equation in fact form a kind of AFBSDDE which is a special case of (1.1). More importantly, the motivation for us to study this kind of stochastic system (1.1) is that it has practical background and can describe more general economic and financial framework with delay. We believe that AFBSDDEs and their wide applications in mathematical finance, if the time delay is allowed, is an important concern in the future.

(ii) The cost functional (1.2) is more general. It involves not only the running cost, the initial cost and the terminal cost, but also delayed terms in the state and control variables.

(iii) Both the necessary and sufficient conditions for the optimal control are obtained. When the control domain is not necessarily convex and the control system described by the fully coupled AFBSDDE, this problem is more difficult. We overcome the difficulties by techniques dealing with fully-coupling in Shi and Wu [21], by methods dealing with time delay in Chen and $\mathrm{Wu}[5]$ and by Clarke generalized gradient's approach dealing with sufficient conditions of optimality in Zhou [30].

We refer to $\mathrm{Wu}$ [25], Shi and $\mathrm{Wu}[21,22]$, Meng [13], Yong [26] for more details on maximum principles for fully coupled forward-backward stochastic systems without delay.

The rest of this paper is organized as follows. In Section 2, we give the main results of this paper, including the necessary and sufficient maximum principles. For the sake of readability, the proofs of the results are spread over several subsections in Section 3. In Section 4, we present two linear-quadratic (LQ) problems as examples of applying the general results established. Finally in Section 5, we give some concluding remarks.

\section{Statement of NECESSARY AND SUfFiCIENT MAXIMUM PRINCIPLES}

In this section, we present the necessary and sufficient maximum principles for our problem (1.3). For readability, proofs of these results will be given in next section.

Let $u(\cdot) \in \mathcal{U}_{a d}$ be optimal, $\Gamma(\cdot) \equiv(x(\cdot), y(\cdot), z(\cdot))$ be the corresponding optimal trajectory corresponding to $u(\cdot)$. For simplification, we introduce the following notations:

$$
\begin{aligned}
b_{x} & :=b_{x}(t, x(t), y(t), z(t), x(t-\delta), u(t), u(t-\delta)), \text { etc. } \\
b\left(u^{\varepsilon}\right) & :=b\left(t, x(t), y(t), z(t), x(t-\delta), u^{\varepsilon}(t), u^{\varepsilon}(t-\delta)\right), b(u):=b(t, x(t), y(t), z(t), x(t-\delta), u(t), u(t-\delta)),
\end{aligned}
$$


and similar notations are used for $\sigma, f, l$. We introduce the following adjoint equation:

$$
\left\{\begin{array}{c}
\mathrm{d} p(t)=\left[f_{y}^{\top} p(t)-b_{y}^{\top} q(t)-\sigma_{y}^{\top} k(t)-l_{y}+f_{y(+\delta)}^{\top}(t-\delta) p(t-\delta)\right] \mathrm{d} t \\
\quad+\left[f_{z}^{\top} p(t)-b_{z}^{\top} q(t)-\sigma_{z}^{\top} k(t)-l_{z}\right] \mathrm{d} W(t), \quad t \in[0, T], \\
-\mathrm{d} q(t)=\left\{-f_{x}^{\top} p(t)+b_{x}^{\top} q(t)+\sigma_{x}^{\top} k(t)+l_{x}+\mathbb{E}^{\mathcal{F}_{t}}\left[\left(\left.b_{x(\delta)}\right|_{t+\delta}\right)^{\top} q(t+\delta)\right.\right. \\
\left.\left.\quad+\left(\left.\sigma_{x(\delta)}\right|_{t+\delta}\right)^{\top} k(t+\delta)+\left.l_{x(\delta)}\right|_{t+\delta}\right]\right\} \mathrm{d} t-k(t) \mathrm{d} W(t), t \in[0, T], \\
p(0)=-\gamma_{y}(y(0)), q(T)=-g_{x}^{\top}(x(T)) p(T)+\Phi_{x}(x(T)), k(T)=0 \\
p(t)=0, \quad t \in[-\delta, 0) ; q(t)=0, k(t)=0, \quad t \in(T, T+\delta] .
\end{array}\right.
$$

Remark 2.1. In equation (2.1), $\left.b_{x(\delta)}\right|_{t+\delta}\left(\right.$ espectively, $\left.\left.\sigma_{x(\delta)}\right|_{t+\delta}\right)$ denotes the value of $b_{x(\delta)}\left(\right.$ espectively, $\left.\sigma_{x(\delta)}\right)$ when time $t$ takes value $t+\delta$. It is easy to see that (2.1) is a linear AFBSDDE and by Lemma 1.1 there exists a unique solution $(p(\cdot), q(\cdot), k(\cdot)) \in L_{\mathcal{F}}^{2}\left([-\delta, T] ; \mathbf{R}^{m}\right) \times L_{\mathcal{F}}^{2}\left([0, T+\delta] ; \mathbf{R}^{n}\right) \times L_{\mathcal{F}}^{2}\left([0, T+\delta] ; \mathbf{R}^{n \times d}\right)$.

Define the Hamiltonian function $H:[0, T] \times \mathbf{R}^{n} \times \mathbf{R}^{m} \times \mathbf{R}^{m \times d} \times \mathbf{R}^{n} \times \mathbf{R}^{m} \times \mathbf{U} \times \mathbf{U} \times \mathbf{R}^{m} \times \mathbf{R}^{n} \times \mathbf{R}^{n \times d} \rightarrow \mathbf{R}$ as

$$
\begin{aligned}
H(t, x, y, z, x(\delta), y(+\delta), v, v(\delta), p, q, k):= & \langle q, b(t, x, y, z, x(\delta), v, v(\delta))\rangle-\langle p, f(t, x, y, z, y(+\delta), v, v(\delta))\rangle \\
& +\operatorname{tr}\left\{k^{\top} \sigma(t, x, y, z, x(\delta))\right\}+l(t, x, y, z, x(\delta), v, v(\delta)) .
\end{aligned}
$$

Then (2.1) can be rewritten as the following stochastic Hamiltonian system's type:

$$
\left\{\begin{array}{l}
\mathrm{d} p(t)=\left[-H_{y}(t)-H_{y(+\delta)}(t-\delta)\right] \mathrm{d} t-H_{z}(t) \mathrm{d} W(t), \quad t \in[0, T], \\
-\mathrm{d} q(t)=\left\{H_{x}(t)+\mathbb{E}^{\mathcal{F}_{t}}\left[\left.H_{x(\delta)}(t)\right|_{t+\delta}\right]\right\} \mathrm{d} t-k(t) \mathrm{d} W(t), \quad t \in[0, T], \\
p(0)=-\gamma_{y}(y(0)), q(T)=-g_{x}^{\top}(x(T)) p(T)+\Phi_{x}(x(T)), k(T)=0, \\
p(t)=0, t \in[-\delta, 0) ; q(t)=0, k(t)=0, t \in(T, T+\delta],
\end{array}\right.
$$

where $H_{x}(t):=H_{x}\left(t, x(t), y(t), z(t), x(t-\delta), \mathbb{E}^{\mathcal{F}_{t}}[y(t+\delta)], u(t), u(t-\delta), p(t), q(t), k(t)\right)$, etc.

The two main results of this paper are the following theorems.

Theorem 2.2 (stochastic maximum principle). Suppose that $(\mathbf{H 1}) \sim(\mathbf{H 3})$ hold. Let $u(\cdot)$ be an optimal control for our problem $(1.3),(x(\cdot), y(\cdot), z(\cdot))$ be the optimal trajectory and $(p(\cdot), q(\cdot), k(\cdot))$ be the solution of adjoint equation (2.1). Then we have

$$
H(t)+\mathbb{E}^{\mathcal{F}_{t}}\left[\left.H(t)\right|_{t+\delta}\right]=\min _{v \in \mathbf{U}}\left\{H(t, v, u(t-\delta))+\mathbb{E}^{\mathcal{F}_{t}}\left[\left.H(t, u(t), v)\right|_{t+\delta}\right]\right\} \text {, a.s. }
$$

Theorem 2.3 (sufficient conditions for optimality). Suppose that $(\mathbf{H 1}) \sim(\mathbf{H} 4)$ hold. Let $u(\cdot)$ be an admissible control, $(x(\cdot), y(\cdot), z(\cdot))$ be the corresponding trajectory and $(p(\cdot), q(\cdot), k(\cdot))$ be the solution of adjoint equation (2.1). Suppose that $H(t, \cdot, \cdot, \cdot, \cdot, \cdot, v, v(\delta), p, q, k)$ is convex and Lipschitz continuous for all $t \in[0, T]$, then $u(\cdot)$ is optimal if it satisfies $(2.4)$.

Proofs of the proceeding two theorems are deferred to Section 3. 


\section{Proof of Theorems 2.1 And 2.2}

This section is devoted to proofs of the two main theorems of this paper: Theorems 2.1 and 2.2. The proofs will be spread over several subsections.

\subsection{Spike variation and prior estimations}

We introduce the spike variation:

$$
u^{\varepsilon}(t):=\left\{\begin{array}{l}
v(t), \text { if } \tau \leq t \leq \tau+\varepsilon \\
u(t), \text { otherwise }
\end{array}\right.
$$

where $0 \leq \tau<T, 0<\varepsilon<\delta$ is sufficiently small, and $v(\cdot) \in \mathbf{U}$ is an arbitrary $\mathcal{F}_{t}$-adapted process satisfying $\sup _{0 \leq t \leq T+\delta} \mathbb{E}|v(t)|^{4}<+\infty$. Obviously, $u^{\varepsilon}(\cdot)$ is admissible. Let $\Gamma^{\varepsilon}(\cdot) \equiv\left(x^{\varepsilon}(\cdot), y^{\varepsilon}(\cdot)\right.$, $\left.z^{\varepsilon}(\cdot)\right)$ be the trajectory corresponding to $u^{\varepsilon}(\cdot)$. We introduce the following variational equation:

$$
\left\{\begin{aligned}
& \mathrm{d} x^{1}(t)= {\left[b_{x} x^{1}(t)+b_{y} y^{1}(t)+b_{z} z^{1}(t)+b_{x(\delta)} x^{1}(t-\delta)+b\left(u^{\varepsilon}\right)-b(u)\right] \mathrm{d} t } \\
&+\left[\sigma_{x} x^{1}(t)+\sigma_{y} y^{1}(t)+\sigma_{z} z^{1}(t)+\sigma_{x(\delta)} x^{1}(t-\delta)\right] \mathrm{d} W(t), \quad t \in[0, T], \\
&-\mathrm{d} y^{1}(t)=\left\{f_{x} x^{1}(t)+f_{y} y^{1}(t)+f_{z} z^{1}(t)+\mathbb{E}^{\mathcal{F}_{t}}\left[f_{y(+\delta)}^{\top} y^{1}(t+\delta)\right]\right. \\
&\left.\quad+f\left(u^{\varepsilon}\right)-f(u)\right\} \mathrm{d} t-z^{1}(t) \mathrm{d} W(t), \quad t \in[0, T] \\
& y^{1}(T)=g_{x}(x(T)) x^{1}(T), \\
& x^{1}(t)=0, t \in[-\delta, 0] ; y^{1}(t)=0, \quad t \in(T, T+\delta] ; z^{1}(t)=0, \quad t \in[T, T+\delta] .
\end{aligned}\right.
$$

Similarly by Lemma 1.1, (3.1) admits a unique adapted solution $\left(x^{1}(\cdot), y^{1}(\cdot), z^{1}(\cdot)\right)$.

The following lemmas in this subsection are all preparations to derive the variational inequality in next subsection. At first, we have the following elementary lemma.

Lemma 3.1. Given $a_{0}(\cdot), b_{01}(\cdot), \ldots, b_{0 d}(\cdot) \in L_{\mathcal{F}}^{2}\left([0, T] ; \mathbf{R}^{n}\right)$ and uniformly bounded $\mathbf{R}^{n \times n}$-valued $\mathcal{F}_{t}$-adapted processes $a_{1}(\cdot), a_{2}(\cdot), b_{11}(\cdot), \ldots, b_{1 d}(\cdot), b_{21}(\cdot), \ldots, b_{2 d}(\cdot)$. Then for the following linear SDDE

$$
\left\{\begin{aligned}
d \tilde{x}(t)= & {\left[a_{1}(t) \tilde{x}(t)+a_{2}(t) \tilde{x}(t-\delta)+a_{0}(t)\right] \mathrm{d} t } \\
& +\sum_{j=1}^{d}\left[b_{1 j}(t) \tilde{x}(t)+b_{2 j}(t) \tilde{x}(t-\delta)+b_{0 j}(t)\right] \mathrm{d} W_{j}(t), t \in[0, T], \\
\tilde{x}(t)= & 0, t \in[-\delta, 0],
\end{aligned}\right.
$$

there exists a constant $K_{1}>0$, such that the unique solution $\tilde{x}(\cdot) \in L_{\mathcal{F}}^{2}\left([-\delta, T] ; \mathbf{R}^{n}\right)$ satisfies

$$
\mathbb{E}\left[\sup _{0 \leq t \leq T}|\tilde{x}(t)|^{2}\right] \leq K_{1}\left\{\int_{0}^{T} \mathbb{E}\left|a_{0}(t)\right|^{2} \mathrm{~d} t+\int_{0}^{T} \mathbb{E}\left|b_{0}(t)\right|^{2} \mathrm{~d} t\right\},
$$

where $b_{0}(\cdot) \equiv\left(b_{01}(\cdot), \ldots, b_{0 d}(\cdot)\right)$.

Given $\xi(\cdot) \in L^{2}\left([T, T+\delta] ; \mathbf{R}^{m}\right), \alpha_{3}(\cdot) \in L_{\mathcal{F}}^{2}\left([0, T] ; \mathbf{R}^{m}\right)$ and uniformly bounded $\mathbf{R}^{m \times m}$-valued $\mathcal{F}_{t}$-adapted processes $\alpha_{0}(\cdot), \alpha_{1}(\cdot), \alpha_{21}(\cdot), \ldots, \alpha_{2 d}(\cdot)$. Then for the following linear ABSDE

$$
\left\{\begin{array}{l}
-\mathrm{d} \tilde{y}(t)=\left[\alpha_{0}(t) \tilde{y}(t)+\alpha_{1}(t) \mathbb{E}^{\mathcal{F}_{t}}[\tilde{y}(t+\delta)]+\sum_{j=1}^{d} \alpha_{2 j}(t) \tilde{z}_{j}(t)+\alpha_{3}(t)\right] \mathrm{d} t-\tilde{z}(t) \mathrm{d} W(t), t \in[0, T], \\
\tilde{y}(t)=\xi(t), t \in[T, T+\delta],
\end{array}\right.
$$


there exists a constant $K_{2}>0$, such that the unique solution $(\tilde{y}(\cdot), \tilde{z}(\cdot)) \in L_{\mathcal{F}}^{2}\left([0, T+\delta] ; \mathbf{R}^{m}\right) \times L_{\mathcal{F}}^{2}\left([0, T] ; \mathbf{R}^{m \times d}\right)$ satisfies

$$
\mathbb{E}\left[\sup _{0 \leq t \leq T}|\tilde{y}(t)|^{2}\right] \leq K_{2}\left\{\mathbb{E}|\xi(T)|^{2}+\int_{0}^{T} \mathbb{E}|\tilde{z}(t)|^{2} \mathrm{~d} t+\int_{0}^{T} \mathbb{E}\left|\alpha_{3}(t)\right|^{2} \mathrm{~d} t+\int_{T}^{T+\delta} \mathbb{E}|\xi(t)|^{2} \mathrm{~d} t\right\},
$$

where $\tilde{z}(\cdot) \equiv\left(\tilde{z}_{1}(\cdot), \ldots, \tilde{z}_{d}(\cdot)\right)$.

Proof. The existence of unique solution of SDDE (3.2) follows from Mohammed [14]. By (3.2) we can get

$$
\begin{aligned}
\sup _{0 \leq t \leq T}|\tilde{x}(t)|^{2} \leq & C\left(\int_{0}^{T}|\tilde{x}(s)|^{2} \mathrm{~d} s+\int_{0}^{T}|\tilde{x}(s-\delta)|^{2} \mathrm{~d} s+\int_{0}^{T}\left|a_{0}(s)\right|^{2} \mathrm{~d} s\right) \\
& +C \sup _{0 \leq t \leq T}\left\{\sum_{j=1}^{d} \int_{0}^{t}\left[b_{1 j}(s) \tilde{x}(s)+b_{2 j}(s) \tilde{x}(s-\delta)+b_{0 j}(s)\right] \mathrm{d} W_{j}(s)\right\}^{2} .
\end{aligned}
$$

Taking expectation on both sides and by Burkholder-Davis-Gundy's inequality, we have

$$
\begin{aligned}
\mathbb{E}\left[\sup _{0 \leq t \leq T}|\tilde{x}(t)|^{2}\right] \leq & C \mathbb{E}\left\{\int_{0}^{T}|\tilde{x}(s)|^{2} \mathrm{~d} s+\int_{0}^{T}|\tilde{x}(s-\delta)|^{2} \mathrm{~d} s+\int_{0}^{T}\left|a_{0}(s)\right|^{2} \mathrm{~d} s+\sum_{j=1}^{d} \int_{0}^{t} \mid b_{1 j}(s) \tilde{x}(s)\right. \\
& \left.+b_{2 j}(s) \tilde{x}(s-\delta)+\left.b_{0 j}(s)\right|^{2} \mathrm{~d} s\right\} \\
\leq & C\left\{\int_{0}^{T} \mathbb{E}|\tilde{x}(s)|^{2} \mathrm{~d} s+\int_{0}^{T} \mathbb{E}|\tilde{x}(s-\delta)|^{2} \mathrm{~d} s+\int_{0}^{T} \mathbb{E}\left|a_{0}(s)\right|^{2} \mathrm{~d} s\right. \\
& \left.+\sum_{j=1}^{d} \int_{0}^{T} \mathbb{E}\left|b_{0 j}(s)\right|^{2} \mathrm{~d} s\right\} .
\end{aligned}
$$

Noting that

$$
\int_{0}^{T} \mathbb{E}|\tilde{x}(s-\delta)|^{2} \mathrm{~d} s=\int_{-\delta}^{T-\delta} \mathbb{E}|\tilde{x}(s)|^{2} \mathrm{~d} s=\int_{0}^{T-\delta} \mathbb{E}|\tilde{x}(s)|^{2} \mathrm{~d} s \leq \int_{0}^{T} \mathbb{E}|\tilde{x}(s)|^{2} \mathrm{~d} s,
$$

then we have

$$
\mathbb{E}\left[\sup _{0 \leq t \leq T}|\tilde{x}(t)|^{2}\right] \leq C \int_{0}^{T} \mathbb{E}\left[\sup _{0 \leq s \leq t}|\tilde{x}(s)|^{2}\right] \mathrm{d} t+C\left\{\int_{0}^{T} \mathbb{E}\left|a_{0}(s)\right|^{2} \mathrm{~d} s+\sum_{j=1}^{d} \int_{0}^{T} \mathbb{E}\left|b_{0 j}(s)\right|^{2} \mathrm{~d} s\right\} .
$$

By Gronwall's inequality, we obtain (3.3). The existence of unique solution of ABSDE (3.4) follows from Peng and Yang [20]. And by (3.4), we can get

$$
\begin{aligned}
\sup _{0 \leq t \leq T}|\tilde{y}(t)|^{2} \leq & C|\xi(T)|^{2}+C \int_{0}^{T}\left\{|\tilde{y}(s)|^{2}+\left|\mathbb{E}^{\mathcal{F}_{s}}[\tilde{y}(s+\delta)]\right|^{2}+\sum_{j=1}^{d}\left|\tilde{z}_{j}(s)\right|^{2}+\left|\alpha_{3}(s)\right|^{2}\right\} \mathrm{d} s \\
& +C\left(\int_{0}^{T} \tilde{z}(s) \mathrm{d} W(s)\right)^{2}+C \sup _{0 \leq t \leq T}\left(\int_{0}^{t} \tilde{z}(s) \mathrm{d} W(s)\right)^{2} .
\end{aligned}
$$


Taking expectation on both sides and by Burkholder-Davis-Gundy's inequality, we have

$$
\begin{aligned}
\mathbb{E}\left[\sup _{0 \leq t \leq T}|\tilde{y}(t)|^{2}\right] \leq & C \mathbb{E}|\xi(T)|^{2}+C\left\{\int_{0}^{T} \mathbb{E}|\tilde{y}(s)|^{2} \mathrm{~d} s+\int_{0}^{T} \mathbb{E}\left|\mathbb{E}^{\mathcal{F}_{s}}[\tilde{y}(s+\delta)]\right|^{2} \mathrm{~d} s+\sum_{j=1}^{d} \int_{0}^{T} \mathbb{E}\left|\tilde{z}_{j}(s)\right|^{2} \mathrm{~d} s\right\} \\
& +C \int_{0}^{T} \mathbb{E}\left|\alpha_{3}(s)\right|^{2} \mathrm{~d} s .
\end{aligned}
$$

Noting that

$$
\begin{aligned}
\int_{0}^{T} \mathbb{E}\left|\mathbb{E}^{\mathcal{F}_{s}}[\tilde{y}(s+\delta)]\right|^{2} \mathrm{~d} s & \leq \int_{0}^{T} \mathbb{E}\left[\mathbb{E}^{\mathcal{F}_{s}}|\tilde{y}(s+\delta)|^{2}\right] \mathrm{d} s=\int_{0}^{T} \mathbb{E}|\tilde{y}(s+\delta)|^{2} \mathrm{~d} s=\int_{\delta}^{T+\delta} \mathbb{E}|\tilde{y}(s)|^{2} \mathrm{~d} s \\
& =\int_{\delta}^{T} \mathbb{E}|\tilde{y}(s)|^{2} \mathrm{~d} s+\int_{T}^{T+\delta} \mathbb{E}|\xi(s)|^{2} \mathrm{~d} s \leq \int_{0}^{T} \mathbb{E}|\tilde{y}(s)|^{2} \mathrm{~d} s+\int_{T}^{T+\delta} \mathbb{E}|\xi(s)|^{2} \mathrm{~d} s
\end{aligned}
$$

then we have

$$
\begin{aligned}
\mathbb{E}\left[\sup _{0 \leq t \leq T}|\tilde{y}(t)|^{2}\right] \leq & C \mathbb{E}|\xi(T)|^{2}+C\left\{\int_{0}^{T} \mathbb{E}\left[\sup _{0 \leq s \leq t}|\tilde{y}(s)|^{2}\right] \mathrm{d} t+\sum_{j=1}^{d} \int_{0}^{T} \mathbb{E}\left|\tilde{z}_{j}(s)\right|^{2} \mathrm{~d} s\right\} \\
& +C \int_{T}^{T+\delta} \mathbb{E}|\xi(s)|^{2} \mathrm{~d} s+C \int_{0}^{T} \mathbb{E}\left|\alpha_{3}(s)\right|^{2} \mathrm{~d} s .
\end{aligned}
$$

By Gronwall's inequality, we obtain (3.5). The proof is complete.

We need the following lemma.

Lemma 3.2. Let (H1) and (H2) hold. Then, we have the following estimations:

$$
\begin{aligned}
& \sup _{0 \leq t \leq T} \mathbb{E}\left|x^{1}(t)\right|^{2} \leq C \varepsilon, \\
& \sup _{0 \leq t \leq T} \mathbb{E}\left|y^{1}(t)\right|^{2} \leq C \varepsilon, \quad \mathbb{E} \int_{0}^{T}\left|z^{1}(t)\right|^{2} \mathrm{~d} t \leq C \varepsilon .
\end{aligned}
$$

Proof. Applying Itô's Formula to $\left\langle G x^{1}(\cdot), y^{1}(\cdot)\right\rangle$, we can get

$$
\begin{aligned}
\mathbb{E}\left\langle g_{x}(x(T)) x^{1}(T), G x^{1}(T)\right\rangle \\
=-\mathbb{E} \int_{0}^{T}\left\langle f_{x} x^{1}(t)+f_{y} y^{1}(t)+f_{z} z^{1}(t)+\mathbb{E}^{\mathcal{F}_{t}}\left[f_{y(+\delta)} y^{1}(t+\delta)\right], G x^{1}(t)\right\rangle \mathrm{d} t-\mathbb{E} \int_{0}^{T}\left\langle f\left(u^{\varepsilon}\right)-f(u), G x^{1}(t)\right\rangle \mathrm{d} t \\
+\mathbb{E} \int_{0}^{T}\left\langle b_{x} x^{1}(t)+b_{y} y^{1}(t)+b_{z} z^{1}(t)+b_{x(\delta)} x^{1}(t-\delta), G^{\top} y^{1}(t)\right\rangle \mathrm{d} t+\mathbb{E} \int_{0}^{T}\left\langle b\left(u^{\varepsilon}\right)-b(u), G^{\top} y^{1}(t)\right\rangle \mathrm{d} t \\
+\mathbb{E} \int_{0}^{T}\left\langle\sigma_{x}(t) x^{1}(t)+\sigma_{y}(t) y^{1}(t)+\sigma_{z} z^{1}(t)+\sigma_{x(\delta)} x^{1}(t-\delta), G^{\top} z^{1}(t)\right\rangle \mathrm{d} t .
\end{aligned}
$$

Then by the $G$-monotonic condition (H2), we obtain

$$
\begin{aligned}
& \mu_{1} \mathbb{E}\left|G x^{1}(T)\right|^{2}+\beta_{1} \mathbb{E} \int_{0}^{T}\left|G x^{1}(t)\right|^{2} \mathrm{~d} t+\beta_{2} \mathbb{E} \int_{0}^{T}\left(\left|G^{\top} y^{1}(t)\right|^{2}+\left|G^{\top} z^{1}(t)\right|^{2}\right) \mathrm{d} t \\
\leq & \mathbb{E} \int_{0}^{T}\left\langle b\left(u^{\varepsilon}\right)-b(u), G^{\top} y^{1}(t)\right\rangle \mathrm{d} t-\mathbb{E} \int_{0}^{T}\left\langle f\left(u^{\varepsilon}\right)-f(u), G x^{1}(t)\right\rangle \mathrm{d} t .
\end{aligned}
$$


Case 1. When $m>n$, we assume $\beta_{1}>0, \beta_{2} \geq 0, \mu_{1}>0$. From (3.8) we have

$$
\begin{aligned}
\mu_{1} \mathbb{E}\left|G x^{1}(T)\right|^{2}+\beta_{1} \mathbb{E} \int_{0}^{T}\left|G x^{1}(t)\right|^{2} \mathrm{~d} t \leq & \mathbb{E} \int_{0}^{T}\left\langle b\left(u^{\varepsilon}\right)-b(u), G^{\top} y^{1}(t)\right\rangle \mathrm{d} t-\mathbb{E} \int_{0}^{T}\left\langle f\left(u^{\varepsilon}\right)-f(u), G x^{1}(t)\right\rangle \mathrm{d} t \\
\leq & \mathbb{E} \int_{0}^{T}\left|G^{\top} y^{1}(t)\right|^{2} \mathrm{~d} t+\frac{1}{4} \mathbb{E} \int_{0}^{T}\left|b\left(u^{\varepsilon}\right)-b(u)\right|^{2} \mathrm{~d} t \\
& +\frac{\beta_{1}}{2} \mathbb{E} \int_{0}^{T}\left|G x^{1}(t)\right|^{2} \mathrm{~d} t+\frac{1}{2 \beta_{1}} \mathbb{E} \int_{0}^{T}\left|f\left(u^{\varepsilon}\right)-f(u)\right|^{2} \mathrm{~d} t,
\end{aligned}
$$

i.e.,

$$
\mu_{1} \mathbb{E}\left|G x^{1}(T)\right|^{2}+\frac{\beta_{1}}{2} \mathbb{E} \int_{0}^{T}\left|G x^{1}(t)\right|^{2} \mathrm{~d} t \leq \mathbb{E} \int_{0}^{T}\left|G^{\top} y^{1}(t)\right|^{2} \mathrm{~d} t+C \varepsilon,
$$

where constant $C$ depends on Lipschitz constants. From the second equation of (3.1), we get

$$
\begin{aligned}
& \mathbb{E}\left|y^{1}(t)\right|^{2}+\mathbb{E} \int_{t}^{T}\left|z^{1}(s)\right|^{2} \mathrm{~d} s \\
= & \left.\mathbb{E}\left\{h_{x}(x(T)) x^{1}(T)+\int_{t}^{T}\left[f_{x} x^{1}(s)+f_{y} y^{1}(s)+f_{z} z^{1}(s)+\mathbb{E}^{\mathcal{F}_{s}}\left[f_{y(+\delta)} y^{1}(s+\delta)\right]+f\left(u^{\varepsilon}\right)-f(u)\right] \mathrm{d} s\right\}\right\}^{2} \\
\leq & 7 C^{2}\left\{\mathbb{E}\left|x^{1}(T)\right|^{2}+T \mathbb{E} \int_{t}^{T}\left|x^{1}(s)\right|^{2} \mathrm{~d} s+T \int_{t}^{T} \mathbb{E}\left|y^{1}(s)\right|^{2} \mathrm{~d} s+(T-t) \mathbb{E} \int_{t}^{T}\left|z^{1}(s)\right|^{2} \mathrm{~d} s\right. \\
& \left.+T \int_{t}^{T} \mathbb{E}\left|\mathbb{E}^{\mathcal{F}_{s}}\left[y^{1}(s+\delta)\right]\right|^{2} \mathrm{~d} s\right\}+7 \mathbb{E}\left(\int_{t}^{T}\left(f\left(u^{\varepsilon}\right)-f(u)\right) \mathrm{d} s\right)^{2} \\
\leq & C_{1}\left\{\mathbb{E}\left|x^{1}(T)\right|^{2}+T \mathbb{E} \int_{t}^{T}\left(\left|x^{1}(s)\right|^{2}+\left|y^{1}(s)\right|^{2}\right) \mathrm{d} s+(T-t) \mathbb{E} \int_{t}^{T}\left|z^{1}(s)\right|^{2} \mathrm{~d} s+\mathbb{E}\left(\int_{t}^{T}\left(f\left(u^{\varepsilon}\right)-f(u)\right) \mathrm{d} s\right)^{2}\right\},
\end{aligned}
$$

where $C_{1}$ is a constant depending on the following $C_{0}$. In the above we have use the fact that

$$
\begin{aligned}
& \int_{t}^{T} \mathbb{E}\left|\mathbb{E}^{\mathcal{F}_{s}}\left[y^{1}(s+\delta)\right]\right|^{2} \mathrm{~d} s \leq \int_{t}^{T} \mathbb{E}\left[\mathbb{E}^{\mathcal{F}_{s}}\left[\left|y^{1}(s+\delta)\right|^{2}\right]\right] \mathrm{d} s=\int_{t}^{T} \mathbb{E}\left[\left|y^{1}(s+\delta)\right|^{2}\right] \mathrm{d} s=\int_{t-\delta}^{T-\delta} \mathbb{E}\left|y^{1}(s)\right|^{2} \mathrm{~d} s \\
\leq & \int_{t-\delta}^{T} \mathbb{E}\left|y^{1}(s)\right|^{2} \mathrm{~d} s=\int_{t-\delta}^{t} \mathbb{E}\left|y^{1}(s)\right|^{2} \mathrm{~d} s+\int_{t}^{T} \mathbb{E}\left|y^{1}(s)\right|^{2} \mathrm{~d} s \leq C_{0} \int_{t}^{T} \mathbb{E}\left|y^{1}(s)\right|^{2} \mathrm{~d} s,
\end{aligned}
$$

where constant $C_{0}>1$ is large enough satisfying $\left(C_{0}-1\right) \int_{t}^{T} \mathbb{E}\left|y^{1}(s)\right|^{2} \mathrm{~d} s \geq \int_{t-\delta}^{t} \mathbb{E}\left|y^{1}(s)\right|^{2} \mathrm{~d} s, t \in[0, T]$.

Let $\delta_{1}=\frac{1}{2 C_{1}}$, then for $t \in\left[T-\delta_{1}, T\right]$, we have

$$
\begin{aligned}
\mathbb{E}\left|y^{1}(t)\right|^{2}+\frac{1}{2} \mathbb{E} \int_{t}^{T}\left|z^{1}(s)\right|^{2} \mathrm{~d} s \leq & C_{1}\left\{\mathbb{E}\left|x^{1}(T)\right|^{2}+T \mathbb{E} \int_{t}^{T}\left|x^{1}(s)\right|^{2} \mathrm{~d} s+T \int_{t}^{T} \mathbb{E}\left|y^{1}(s)\right|^{2} \mathrm{~d} s\right\} \\
& \left.+\mathbb{E}\left(\int_{t}^{T}\left(f\left(u^{\varepsilon}\right)-f(u)\right) \mathrm{d} s\right)^{2}\right\} .
\end{aligned}
$$

From (3.9), we get

$$
\mathbb{E}\left|y^{1}(t)\right|^{2}+\frac{1}{2} \mathbb{E} \int_{t}^{T}\left|z^{1}(s)\right|^{2} \mathrm{~d} s \leq C_{2} \int_{t}^{T} \mathbb{E}\left|y^{1}(s)\right|^{2} \mathrm{~d} s+C \varepsilon+C \varepsilon^{2},
$$


where $C_{2}$ is a constant depending on $C_{1}, \mu_{1}, \beta_{1}$ and $T$. By the Gronwall's inequality, we have

$$
\mathbb{E}\left|y^{1}(t)\right|^{2} \leq C \varepsilon, \quad \mathbb{E} \int_{t}^{T}\left|z^{1}(s)\right|^{2} \mathrm{~d} s \leq C \varepsilon, \quad t \in\left[T-\delta_{1}, T\right]
$$

Repeating this procedure, the above estimates hold for $t \in\left[T-2 \delta_{1}, T\right]$. Obviously, after a finite number of iterations, estimation (3.7) are obtained. By the variation equation (3.1), estimation (3.7), using the same technique to deal with the time delay term as in the proof of Lemma 3.1, we get

$$
\begin{aligned}
\mathbb{E}\left|x^{1}(t)\right|^{2}= & \mathbb{E}\left\{\int_{0}^{t}\left[b_{x} x^{1}(s)+b_{y} y^{1}(s)+b_{z} z^{1}(s)+b_{x(\delta)} x^{1}(t-\delta)+b\left(u^{\varepsilon}\right)-b(u)\right] \mathrm{d} s\right. \\
& \left.+\int_{0}^{t}\left[\sigma_{x} x^{1}(s)+\sigma_{y} y^{1}(s)+\sigma_{z} z^{1}(s)+\sigma_{x(\delta)} x^{1}(t-\delta)\right] \mathrm{d} W(s)\right\}^{2} \\
\leq & 18 C^{2}\left\{\int_{0}^{t} \mathbb{E}\left|x^{1}(s)\right|^{2} \mathrm{~d} s+T \sup _{0 \leq t \leq T} \mathbb{E}\left|y^{1}(t)\right|^{2}+\mathbb{E} \int_{0}^{t}\left|z^{1}(s)\right|^{2} \mathrm{~d} s\right) \\
& \left.+\mathbb{E}\left(\int_{0}^{t}\left(b\left(u^{\varepsilon}\right)-b(u)\right) \mathrm{d} s\right)^{2}\right\} \leq C_{3} \int_{0}^{t} \mathbb{E}\left|x^{1}(s)\right|^{2} \mathrm{~d} s+C \varepsilon+C \varepsilon^{2},
\end{aligned}
$$

where $C_{3}$ is a constant. By Gronwall's inequality, we obtain (3.6).

Case 2. When $m<n$, we assume $\beta_{1} \geq 0, \beta_{2}>0, \mu_{1} \geq 0$. From (3.8) we have

$$
\begin{aligned}
& \beta_{2} \mathbb{E} \int_{0}^{T}\left(\left|G^{\top} y^{1}(t)\right|^{2}+\left|G^{\top} z^{1}(t)\right|^{2}\right) \mathrm{d} t \\
\leq & \mathbb{E} \int_{0}^{T}\left\langle b\left(u^{\varepsilon}\right)-b(u), G^{\top} y^{1}(t)\right\rangle \mathrm{d} t-\mathbb{E} \int_{0}^{T}\left\langle f\left(u^{\varepsilon}\right)-f(u), G x^{1}(t)\right\rangle \mathrm{d} t \\
\leq & \frac{\beta_{2}}{2} \mathbb{E} \int_{0}^{T}\left|G^{\top} y^{1}(t)\right|^{2} \mathrm{~d} t+\frac{1}{2 \beta_{2}} \mathbb{E} \int_{0}^{T} \mid b\left(u^{\varepsilon}-\left.b(u)\right|^{2} \mathrm{~d} t r+\mathbb{E} \int_{0}^{T}\left|G x^{1}(t)\right|^{2} \mathrm{~d} t+\frac{1}{4} \mathbb{E} \int_{0}^{T}\left|f\left(u^{\varepsilon}\right)-f(u)\right|^{2} \mathrm{~d} t,\right.
\end{aligned}
$$

i.e.,

$$
\frac{\beta_{2}}{2} \mathbb{E} \int_{0}^{T}\left|G^{\top} y^{1}(t)\right|^{2} \mathrm{~d} t+\beta_{2} \mathbb{E} \int_{0}^{T}\left|G^{\top} z^{1}(t)\right|^{2} \mathrm{~d} t \leq \mathbb{E} \int_{0}^{T}\left|G x^{1}(t)\right|^{2} \mathrm{~d} t+C \varepsilon,
$$

where constant $C$ depends on Lipschitz constants and $\beta_{2}$. By (3.1) and (3.10), we get

$$
\begin{aligned}
\mathbb{E}\left|x^{1}(t)\right|^{2} \leq & 9 C^{2}\left\{\int_{0}^{t} \mathbb{E}\left|x^{1}(s)\right|^{2} \mathrm{~d} s+\mathbb{E} \int_{0}^{t}\left|y^{1}(s)\right|^{2} \mathrm{~d} s+\mathbb{E} \int_{0}^{t}\left|z^{1}(s)\right|^{2} \mathrm{~d} s\right. \\
& \left.+\int_{0}^{t} \mathbb{E}\left|x^{1}(s-\delta)\right|^{2} \mathrm{~d} s+\mathbb{E}\left(\int_{0}^{t}\left(b\left(u^{\varepsilon}\right)-b(u)\right) \mathrm{d} s\right)^{2}\right\} \\
\leq & C_{4} \int_{0}^{t} \mathbb{E}\left|x^{1}(s)\right|^{2} \mathrm{~d} s+C \varepsilon+C \varepsilon^{2},
\end{aligned}
$$

where $C_{4}$ is a constant depending on $\beta_{2}, G$. By Gronwall's inequality, estimation (3.6) are obtained. By (3.1), estimation (3.6) and the same technique to deal with the time anticipated term as in Case 1, we get

$$
\mathbb{E}\left|y^{1}(t)\right|^{2}+\mathbb{E} \int_{t}^{T}\left|z^{1}(s)\right|^{2} \mathrm{~d} s \leq C_{5}\left(T \int_{t}^{T} \mathbb{E}\left|y^{1}(s)\right|^{2} \mathrm{~d} s+(T-t) \mathbb{E} \int_{t}^{T}\left|z^{1}(s)\right|^{2} \mathrm{~d} s\right)+C \varepsilon+C \varepsilon^{2},
$$

where constant $C_{5}$ depends on $C_{4}$. Using the above iteration process again, (3.7) is obtained.

Case 3. When $m=n$, similarly to the above two cases, the result can be obtained easily. 
However, the $\varepsilon$-order estimations of $\left(x^{1}(\cdot), y^{1}(\cdot), z^{1}(\cdot)\right)$ in Lemma 3.2 is not sufficient to get the variational inequality. We need to give some more elaborate estimations. Thus we have the following lemma.

Lemma 3.3. Let (H1) and (H2) hold. Then we have

$$
\begin{aligned}
& \sup _{0 \leq t \leq T} \mathbb{E}\left|x^{1}(t)\right|^{2} \leq C \varepsilon^{\frac{3}{2}}, \\
& \sup _{0 \leq t \leq T} \mathbb{E}\left|y^{1}(t)\right|^{2} \leq C \varepsilon^{\frac{3}{2}}, \quad \mathbb{E} \int_{0}^{T}\left|z^{1}(t)\right|^{2} \mathrm{~d} t \leq C \varepsilon^{\frac{3}{2}} .
\end{aligned}
$$

Proof. By Lemmas 3.1 and 3.2, we can easily get

$$
\mathbb{E}\left[\sup _{0 \leq t \leq T}\left|x^{1}(t)\right|^{2}\right] \leq C \varepsilon, \quad \mathbb{E}\left[\sup _{0 \leq t \leq T}\left|y^{1}(t)\right|^{2}\right] \leq C \varepsilon .
$$

By (3.8), Hölder's inequality and (3.13), we have

$$
\begin{aligned}
& \mu_{1} \mathbb{E}\left|G x^{1}(T)\right|^{2}+\beta_{1} \mathbb{E} \int_{0}^{T}\left|G x^{1}(t)\right|^{2} \mathrm{~d} t+\beta_{2} \mathbb{E} \int_{0}^{T}\left(\left|G^{\top} y^{1}(t)\right|^{2}+\left|G^{\top} z^{1}(t)\right|^{2}\right) \mathrm{d} t \\
\leq & \mathbb{E} \int_{0}^{T}\left\langle b\left(u^{\varepsilon}(t)\right)-b(u(t)), G^{\top} y^{1}(t)\right\rangle \mathrm{d} t-\mathbb{E} \int_{0}^{T}\left\langle f\left(u^{\varepsilon}(t)\right)-f(u(t)), G x^{1}(t)\right\rangle \mathrm{d} t \\
\leq & \sqrt{\mathbb{E}\left[\sup _{0 \leq t \leq T}\left|y^{1}(t)\right|^{2}\right] \mathbb{E}\left(\int_{0}^{T}\left|G\left(b\left(u^{\varepsilon}(t)\right)-b(u(t))\right)\right| \mathrm{d} t\right)^{2}} \\
& +\sqrt{\mathbb{E}\left[\sup _{0 \leq t \leq T}\left|x^{1}(t)\right|^{2}\right] \mathbb{E}\left(\int_{0}^{T}\left|G^{\top}\left(f\left(u^{\varepsilon}(t)\right)-f(u(t))\right)\right| \mathrm{d} t\right)^{2}} \leq C \varepsilon^{\frac{3}{2}} .
\end{aligned}
$$

Case 1. When $m>n$, we have $\beta_{1}>0, \beta_{2} \geq 0, \mu_{1}>0$. Then we obtain

$$
\mu_{1} \mathbb{E}\left|G x^{1}(T)\right|^{2}+\beta_{1} \mathbb{E} \int_{0}^{T}\left|G x^{1}(t)\right|^{2} \mathrm{~d} t \leq C \varepsilon^{\frac{3}{2}} .
$$

Using the same method of Lemma 3.2, we can get estimation (3.12). Then (3.11) holds.

Case 2. When $m<n$, we have $\beta_{1} \geq 0, \beta_{2}>0, \mu_{1} \geq 0$. Then we have

$$
\beta_{2} \mathbb{E} \int_{0}^{T}\left(\left|G^{\top} y^{1}(t)\right|^{2}+\left|G^{\top} z^{1}(t)\right|^{2}\right) \mathrm{d} t \leq C \varepsilon^{\frac{3}{2}}
$$

Using the method of Lemma 3.2 once more, we can get estimation (3.11). Then (3.12) holds.

Case 3. When $m=n$, the result can be obtained similarly.

The following lemma plays an important role in deriving the variational inequality.

Lemma 3.4. Let (H1) and (H2) hold. Then we have

$$
\begin{gathered}
\sup _{0 \leq t \leq T} \mathbb{E}\left|x^{\varepsilon}(t)-x(t)-x^{1}(t)\right|^{2} \leq C_{\varepsilon} \varepsilon^{2}, \\
\sup _{0 \leq t \leq T} \mathbb{E}\left|y^{\varepsilon}(t)-y(t)-y^{1}(t)\right|^{2} \leq C_{\varepsilon} \varepsilon^{2}, \quad \mathbb{E} \int_{0}^{T}\left|z^{\varepsilon}(t)-z(t)-z^{1}(t)\right|^{2} \mathrm{~d} t \leq C_{\varepsilon} \varepsilon^{2} .
\end{gathered}
$$

Hereafter $C_{\varepsilon}$ denotes some nonnegative constant such that $C_{\varepsilon} \rightarrow 0$ as $\varepsilon \rightarrow 0$. 
Proof. It is easy to see that

$$
\begin{aligned}
& \int_{0}^{t} b\left(s, \Gamma(s)+\Gamma^{1}(s), x(s-\delta)+x^{1}(s-\delta), u^{\varepsilon}(s), u^{\varepsilon}(s-\delta)\right) \mathrm{d} s \\
& +\int_{0}^{t} \sigma\left(s, \Gamma(s)+\Gamma^{1}(s), x(s-\delta)+x^{1}(s-\delta)\right) \mathrm{d} W(s) \\
= & \int_{0}^{t}\left[b\left(u^{\varepsilon}\right)+\int_{0}^{1} b_{x}\left(s, \Gamma(s)+\lambda \Gamma^{1}(s), x(s-\delta)+\lambda x^{1}(s-\delta), u^{\varepsilon}(s), u^{\varepsilon}(s-\delta)\right) \mathrm{d} \lambda x^{1}(s)\right. \\
& +\int_{0}^{1} b_{y}\left(s, \Gamma(s)+\lambda \Gamma^{1}(s), x(s-\delta)+\lambda x^{1}(s-\delta), u^{\varepsilon}(s), u^{\varepsilon}(s-\delta)\right) \mathrm{d} \lambda y^{1}(s) \\
& +\int_{0}^{1} b_{z}\left(s, \Gamma(s)+\lambda \Gamma^{1}(s), x(s-\delta)+\lambda x^{1}(s-\delta), u^{\varepsilon}(s), u^{\varepsilon}(s-\delta)\right) \mathrm{d} \lambda z^{1}(s) \\
& \left.+\int_{0}^{1} b_{x_{\delta}}\left(s, \Gamma(s)+\lambda \Gamma^{1}(s), x(s-\delta)+\lambda x^{1}(s-\delta), u^{\varepsilon}(s), u^{\varepsilon}(s-\delta)\right) \mathrm{d} \lambda x^{1}(s-\delta)\right] \mathrm{d} s \\
& +\int_{0}^{t}\left[\sigma(s, \Gamma(s), x(s-\delta))+\int_{0}^{1} \sigma_{x}\left(s, \Gamma(s)+\lambda \Gamma^{1}(s), x(s-\delta)+\lambda x^{1}(s-\delta)\right) \mathrm{d} \lambda x^{1}(s)\right. \\
& +\int_{0}^{1} \sigma_{y}\left(s, \Gamma(s)+\lambda \Gamma^{1}(s), x(s-\delta)+\lambda x^{1}(s-\delta)\right) \mathrm{d} \lambda y^{1}(s) \\
& +\int_{0}^{1} \sigma_{z}\left(s, \Gamma(s)+\lambda \Gamma^{1}(s), x(s-\delta)+\lambda x^{1}(s-\delta)\right) \mathrm{d} \lambda z^{1}(s) \\
& \left.+\int_{0}^{1} \sigma_{x_{\delta}}\left(s, \Gamma(s)+\lambda \Gamma^{1}(s), x(s-\delta)+\lambda x^{1}(s-\delta)\right) \mathrm{d} \lambda x^{1}(s-\delta)\right] \mathrm{d} W(s) \\
= & \int_{0}^{t} b(u) \mathrm{d} s+\int_{0}^{t} \sigma(s, \Gamma(s), x(s-\delta)) \mathrm{d} W(s) \\
& +\int_{0}^{t}\left[b_{x} x^{1}(s)+b_{y} y^{1}(s)+b_{z} z^{1}(s)+b_{x_{\delta}} x^{1}(s-\delta)+b\left(u^{\varepsilon}\right)-b(u)\right] \mathrm{d} s \\
& +\int_{0}^{t}\left[\sigma_{x} x^{1}(s)+\sigma_{y} y^{1}(s)+\sigma_{z} z^{1}(s)+\sigma_{x_{\delta}} x^{1}(s-\delta)\right] \mathrm{d} W(s)+\int_{0}^{t} A_{1}^{\varepsilon}(s) \mathrm{d} s+\int_{0}^{t} B_{1}^{\varepsilon}(s) \mathrm{d} W(s) \\
& \\
& =x^{1}(t)+\int_{0}^{t} A_{1}^{\varepsilon}(s) \mathrm{d} s+\int_{0}^{\varepsilon}(s) \mathrm{d} W(s), \\
&
\end{aligned}
$$

where (for simplification we omit the time subscript $s$ )

$$
\begin{aligned}
A_{1}^{\varepsilon}:= & \int_{0}^{1}\left[b_{x}\left(\Gamma+\lambda \Gamma^{1}, x(\delta)+\lambda x^{1}(\delta), u^{\varepsilon}, u^{\varepsilon}(\delta)\right)-b_{x}\right] \mathrm{d} \lambda x^{1}+\int_{0}^{1}\left[b_{y}\left(\Gamma+\lambda \Gamma^{1}, x(\delta)+\lambda x^{1}(\delta), u^{\varepsilon}, u^{\varepsilon}(\delta)\right)-b_{x}\right] \mathrm{d} \lambda y^{1} \\
& +\int_{0}^{1}\left[b_{z}\left(\Gamma+\lambda \Gamma^{1}, x(\delta)+\lambda x^{1}(\delta), u^{\varepsilon}, u^{\varepsilon}(\delta)\right)-b_{x}\right] \mathrm{d} \lambda z^{1} \\
& +\int_{0}^{1}\left[b_{x(\delta)}\left(\Gamma+\lambda \Gamma^{1}, x(\delta)+\lambda x^{1}(\delta), u^{\varepsilon}, u^{\varepsilon}(\delta)\right)-b_{x}\right] \mathrm{d} \lambda x^{1}(\delta), \\
B_{1}^{\varepsilon}:= & \int_{0}^{1}\left[\sigma_{x}\left(\Gamma+\lambda \Gamma^{1}, x(\delta)+\lambda x^{1}(\delta)\right)-\sigma_{x}\right] \mathrm{d} \lambda x^{1}+\int_{0}^{1}\left[\sigma_{y}\left(\Gamma+\lambda \Gamma^{1}, x(\delta)+\lambda x^{1}(\delta)\right)-\sigma_{x}\right] \mathrm{d} \lambda y^{1} \\
& +\int_{0}^{1}\left[\sigma_{z}\left(\Gamma+\lambda \Gamma^{1}, x(\delta)+\lambda x^{1}(\delta)\right)-\sigma_{x}\right] \mathrm{d} \lambda z^{1}+\int_{0}^{1}\left[\sigma_{x(\delta)}\left(\Gamma+\lambda \Gamma^{1}, x(\delta)+\lambda x^{1}(\delta)\right)-\sigma_{x}\right] \mathrm{d} \lambda x^{1}(\delta),
\end{aligned}
$$


and $\Gamma+\lambda \Gamma^{1} \equiv\left(x+\lambda x^{1}, y+\lambda y^{1}, z+\lambda z^{1}\right)$. By Lemma 3.3, we can easily get

$$
\sup _{0 \leq t \leq T} \mathbb{E}\left\{\left(\int_{0}^{t} A_{1}^{\varepsilon}(s) \mathrm{d} s\right)^{2}+\left(\int_{0}^{t} B_{1}^{\varepsilon}(s) \mathrm{d} W(s)\right)^{2}\right\} \leq C_{\varepsilon} \varepsilon^{2} .
$$

Since

$$
x^{\varepsilon}(t)=x(0)+\int_{0}^{t} b\left(s, \Gamma^{\varepsilon}(s), x^{\varepsilon}(s-\delta), u^{\varepsilon}(s), u^{\varepsilon}(s-\delta)\right) \mathrm{d} s+\int_{0}^{t} \sigma\left(s, \Gamma^{\varepsilon}(s), x^{\varepsilon}(s-\delta)\right) \mathrm{d} W(s),
$$

then

$$
\begin{aligned}
& x^{\varepsilon}(t)-x(t)-x^{1}(t) \\
= & \int_{0}^{t}\left[b\left(s, \Gamma^{\varepsilon}(s), x^{\varepsilon}(s-\delta), u^{\varepsilon}(s), u^{\varepsilon}(s-\delta)\right)-b\left(s, \Gamma(s)+\Gamma^{1}(s), x(s-\delta)+x^{1}(s-\delta), u^{\varepsilon}(s), u^{\varepsilon}(s-\delta)\right)\right] \mathrm{d} s \\
& +\int_{0}^{t}\left[\sigma\left(s, \Gamma^{\varepsilon}(s), x^{\varepsilon}(s-\delta)\right)-\sigma\left(s, \Gamma(s)+\Gamma^{1}(s), x(s-\delta)+x^{1}(s-\delta)\right)\right] \mathrm{d} W(s) \\
& +\int_{0}^{t} A_{1}^{\varepsilon}(s) \mathrm{d} s+\int_{0}^{t} B_{1}^{\varepsilon}(s) \mathrm{d} W(s) \\
= & \int_{0}^{t}\left[I_{1}^{\varepsilon}(s)\left(x^{\varepsilon}(s)-x(s)-x^{1}(s)\right)+I_{2}^{\varepsilon}(s)\left(y^{\varepsilon}(s)-y(s)-y^{1}(s)\right)+I_{3}^{\varepsilon}(s)\left(z^{\varepsilon}(s)-z(s)-z^{1}(s)\right)\right. \\
& \left.+I_{4}^{\varepsilon}(s)\left(x^{\varepsilon}(s-\delta)-x(s-\delta)-x^{1}(s-\delta)\right)\right] \mathrm{d} s+\int_{0}^{t}\left[D_{1}^{\varepsilon}(s)\left(x^{\varepsilon}(s)-x(s)-x^{1}(s)\right)\right. \\
& +D_{2}^{\varepsilon}(s)\left(y^{\varepsilon}(s)-y(s)-y^{1}(s)\right)+D_{3}^{\varepsilon}(s)\left(z^{\varepsilon}(s)-z(s)-z^{1}(s)\right) \\
& \left.+D_{4}^{\varepsilon}(s)\left(x^{\varepsilon}(s-\delta)-x(s-\delta)-x^{1}(s-\delta)\right)\right] \mathrm{d} W(s)+\int_{0}^{t} A_{1}^{\varepsilon}(s) \mathrm{d} s+\int_{0}^{t} B_{1}^{\varepsilon}(s) \mathrm{d} W(s),
\end{aligned}
$$

where

$$
\begin{aligned}
I_{1}^{\varepsilon} & :=\int_{0}^{1} b_{x}\left(\Gamma+\Gamma^{1}+\lambda\left(\Gamma^{\varepsilon}-\Gamma-\Gamma^{1}\right), x(\delta)+x^{1}(\delta)+\lambda\left(x^{\varepsilon}-x(\delta)-x^{1}(\delta)\right), u^{\varepsilon}, u^{\varepsilon}(\delta)\right) \mathrm{d} \lambda, \text { etc. }, \\
D_{1}^{\varepsilon} & :=\int_{0}^{1}\left[\sigma_{x}\left(\Gamma+\Gamma^{1}+\lambda\left(\Gamma^{\varepsilon}-\Gamma-\Gamma^{1}\right), x(\delta)+x^{1}(\delta)+\lambda\left(x^{\varepsilon}-x(\delta)-x^{1}(\delta)\right)\right) \mathrm{d} \lambda,\right. \text { etc. }
\end{aligned}
$$

and $\Gamma+\Gamma^{1}+\lambda\left(\Gamma^{\varepsilon}-\Gamma-\Gamma^{1}\right) \equiv\left(x+x^{1}+\lambda\left(x^{\varepsilon}-x-x^{1}\right), y+y^{1}+\lambda\left(y^{\varepsilon}-y-y^{1}\right), z+z^{1}+\lambda\left(z^{\varepsilon}-z-z^{1}\right)\right)$.

Consequently, by (3.16) and similar technique to deal with the time delay term as in Lemma 3.1, we get

$$
\begin{aligned}
\mathbb{E}\left|x^{\varepsilon}(t)-x(t)-x^{1}(t)\right|^{2} \leq & C \int_{0}^{t}\left(\mathbb{E}\left|x^{\varepsilon}(s)-x(s)-x^{1}(s)\right|^{2}+\mathbb{E}\left|y^{\varepsilon}(s)-y(s)-y^{1}(s)\right|^{2}\right) \mathrm{d} s \\
& +C \mathbb{E} \int_{0}^{t}\left|z^{\varepsilon}(s)-z(s)-z^{1}(s)\right|^{2} \mathrm{~d} s+C_{\varepsilon} \varepsilon^{2} .
\end{aligned}
$$

Moreover, we have

$$
\begin{aligned}
& -\int_{t}^{T} f\left(s, \Gamma(s)+\Gamma^{1}(s), \mathbb{E}^{\mathcal{F}_{s}}\left[y(s+\delta)+y^{1}(s+\delta)\right], u^{\varepsilon}(s), u^{\varepsilon}(s-\delta)\right) \mathrm{d} s+\int_{t}^{T}\left(z(s)+z^{1}(s)\right) \mathrm{d} W(s) \\
= & g(x(T))+g_{x}(x(T)) x^{1}(T)-y(t)-y^{1}(t)-\int_{t}^{T} I_{5}^{\varepsilon}(s) \mathrm{d} s,
\end{aligned}
$$


where

$$
\begin{aligned}
I_{5}^{\varepsilon}:= & \int_{0}^{1}\left[f_{x}\left(\Gamma+\lambda \Gamma^{1}, y(+\delta)+\lambda y^{1}(+\delta), u^{\varepsilon}, u^{\varepsilon}(\delta)\right)-f_{x}\right] \mathrm{d} \lambda x^{1} \\
& +\int_{0}^{1}\left[f_{y}\left(\Gamma+\lambda \Gamma^{1}, y(+\delta)+\lambda y^{1}(+\delta), u^{\varepsilon}, u^{\varepsilon}(\delta)\right)-f_{y}\right] \mathrm{d} \lambda y^{1} \\
& +\int_{0}^{1}\left[f_{z}\left(\Gamma+\lambda \Gamma^{1}, y_{+\delta}+\lambda y_{+\delta}^{1}, u^{\varepsilon}, u^{\varepsilon}(\delta)\right)-f_{z}\right] \mathrm{d} \lambda z^{1} \\
& +\int_{0}^{1}\left[f_{y(+\delta)}\left(\Gamma+\lambda \Gamma^{1}, y(+\delta)+\lambda y^{1}(+\delta), u^{\varepsilon}, u^{\varepsilon}(\delta)\right)-f_{y(+\delta)}\right] \mathrm{d} \lambda y^{1}(+\delta) .
\end{aligned}
$$

By Lemma 3.3, we can easily find

$$
\sup _{0 \leq t \leq T} \mathbb{E}\left(\int_{t}^{T} I_{5}^{\varepsilon}(s) \mathrm{d} s\right)^{2} \leq C_{\varepsilon} \varepsilon^{2}
$$

Then from

$$
y^{\varepsilon}(t)=g\left(x^{\varepsilon}(T)\right)+\int_{t}^{T} f\left(s, \Gamma^{\varepsilon}(s), \mathbb{E}^{\mathcal{F}_{s}}\left[y^{\varepsilon}(s+\delta)\right], u^{\varepsilon}(s), u^{\varepsilon}(s-\delta)\right) \mathrm{d} s-\int_{t}^{T} z^{\varepsilon}(s) \mathrm{d} W(s),
$$

we have

$$
\begin{aligned}
& {\left[y^{\varepsilon}(t)-y(t)-y^{1}(t)\right]+\int_{t}^{T}\left[z^{\varepsilon}(s)-z(s)-z^{1}(s)\right] \mathrm{d} W(s) } \\
= & \int_{t}^{T}\left\{f\left(s, \Gamma^{\varepsilon}(s), \mathbb{E}^{\mathcal{F}_{s}}\left[y^{\varepsilon}(s+\delta)\right], u^{\varepsilon}(s), u^{\varepsilon}(s-\delta)\right)-f\left(s, \Gamma(s)+\Gamma^{1}(s), \mathbb{E}^{\mathcal{F}_{s}}\left[y(s+\delta)+y^{1}(s+\delta)\right],\right.\right. \\
& \left.\left.u^{\varepsilon}(s), u^{\varepsilon}(s-\delta)\right)\right\} \mathrm{d} s+\int_{t}^{T} I_{5}^{\varepsilon}(s) \mathrm{d} s+g\left(x^{\varepsilon}(T)\right)-g(x(T))-g_{x}(x(T)) x^{1}(T) \\
= & \int_{t}^{T}\left\{H_{1}^{\varepsilon}(s)\left(x^{\varepsilon}(s)-x(s)-x^{1}(s)\right)+H_{2}^{\varepsilon}(s)\left(y^{\varepsilon}(s)-y(s)-y^{1}(s)\right)+H_{3}^{\varepsilon}(s)\left(z^{\varepsilon}(s)-z(s)-z^{1}(s)\right)\right. \\
& \left.+H_{4}^{\varepsilon}(s) \mathbb{E}^{\mathcal{F}_{s}}\left[y^{\varepsilon}(s+\delta)-y(s+\delta)-y^{1}(s+\delta)\right]\right\} \mathrm{d} s+\int_{t}^{T} I_{5}^{\varepsilon}(s) \mathrm{d} s \\
& +g\left(x^{\varepsilon}(T)\right)-g\left(x(T)+x^{1}(T)\right)+\int_{0}^{1}\left[g_{x}\left(x(T)+\lambda x^{1}(T)\right)-g_{x}(x(T))\right] \mathrm{d} \lambda x^{1}(T),
\end{aligned}
$$

where

$$
H_{1}^{\varepsilon}:=\int_{0}^{1} f_{x}\left(\Gamma+\Gamma^{1}+\lambda\left(\Gamma^{\varepsilon}-\Gamma-\Gamma^{1}\right), \mathbb{E}^{\mathcal{F}_{s}}\left[y(+\delta)+y^{1}(+\delta)+\lambda\left(y^{\varepsilon}-y(+\delta)-y^{1}(+\delta)\right), u^{\varepsilon}, u^{\varepsilon}(\delta)\right) \mathrm{d} \lambda,\right. \text { etc. }
$$

Immediately the following result holds:

$$
\begin{aligned}
& \mathbb{E}\left|y^{\varepsilon}(t)-y(t)-y^{1}(t)\right|^{2}+\mathbb{E} \int_{t}^{T}\left|z^{\varepsilon}(s)-z(s)-z^{1}(s)\right|^{2} \mathrm{~d} s \\
\leq & C \mathbb{E}\left\{\int _ { t } ^ { T } \left[H_{1}^{\varepsilon}(s)\left(x^{\varepsilon}(s)-x(s)-x^{1}(s)\right)+H_{2}^{\varepsilon}(s)\left(y^{\varepsilon}(s)-y(s)-y^{1}(s)\right)\right.\right. \\
& \left.+H_{3}^{\varepsilon}(s)\left(z^{\varepsilon}(s)-z(s)-z^{1}(s)\right)+H_{4}^{\varepsilon}(s) \mathbb{E}^{\mathcal{F}_{s}}\left[y^{\varepsilon}(s+\delta)-y(s+\delta)-y^{1}(s+\delta)\right]\right] \mathrm{d} s \\
& \left.+\int_{t}^{T} I_{5}^{\varepsilon}(s) \mathrm{d} s+g\left(x^{\varepsilon}(T)\right)-g\left(x(T)+x^{1}(T)\right)+\int_{0}^{1}\left[g_{x}\left(x(T)+\lambda x^{1}(T)\right)-g_{x}(x(T))\right] \mathrm{d} \lambda x^{1}(T)\right\}^{2} .
\end{aligned}
$$


By (3.18), Lemma 3.3, the same iteration method and the technique to deal with the time anticipated term as in Lemma 3.2, we have

$$
\begin{aligned}
& \mathbb{E}\left|y^{\varepsilon}(t)-y(t)-y^{1}(t)\right|^{2}+\frac{1}{2} \mathbb{E} \int_{t}^{T}\left|z^{\varepsilon}(s)-z(s)-z^{1}(s)\right|^{2} \mathrm{~d} s \leq C \int_{t}^{T}\left(\mathbb{E}\left|x^{\varepsilon}(s)-x(s)-x^{1}(s)\right|^{2}\right. \\
& \left.+\mathbb{E}\left|y^{\varepsilon}(s)-y(s)-y^{1}(s)\right|^{2}\right) \mathrm{d} s+\mathbb{E}\left|h\left(x^{\varepsilon}(T)\right)-h\left(x(T)+x^{1}(T)\right)\right|^{2}+C_{\varepsilon} \varepsilon^{2} .
\end{aligned}
$$

From (3.17) and (3.19), using the method once more as the proof of Lemma 3.3 with $\left(x^{1}(\cdot), y^{1}(\cdot), z^{1}(\cdot)\right)$ replaced by $\left(x^{\varepsilon}(\cdot)-x(\cdot)-x^{1}(\cdot), y^{\varepsilon}(\cdot)-y(\cdot)-y^{1}(\cdot), z^{\varepsilon}(\cdot)-z(\cdot)-z^{1}(\cdot)\right)$, we can get (3.14) and (3.15). We omit the detail. The proof is complete.

\subsection{Variational inequality}

Lemma 3.5. Let $(\mathbf{H 1}) \sim(\mathbf{H} 3)$ hold. Then we have

$$
\begin{aligned}
& \mathbb{E} \int_{0}^{T}\left[l_{x}^{\top} x^{1}(t)+l_{y}^{\top} y^{1}(t)+l_{z}^{\top} x^{1}(t)+l_{x(\delta)}^{\top} x^{1}(t-\delta)+l\left(u^{\varepsilon}\right)-l(u)\right] \mathrm{d} t \\
& +\mathbb{E}\left[\Phi_{x}^{\top}(x(T)) x^{1}(T)\right]+\mathbb{E}\left[\gamma_{y}^{\top}(y(0)) y^{1}(0)\right] \geq o(\varepsilon) .
\end{aligned}
$$

Proof. Since $u(\cdot)$ is optimal, we have

$$
\begin{aligned}
0 \leq J\left(u^{\varepsilon}(\cdot)\right)-J(u(\cdot))= & \mathbb{E} \int_{0}^{T}\left[l\left(t, x^{\varepsilon}(t), y^{\varepsilon}(t), z^{\varepsilon}(t), x^{\varepsilon}(t-\delta), u^{\varepsilon}(t), u^{\varepsilon}(t-\delta)\right)-l(u)\right] \mathrm{d} t \\
& +\mathbb{E}\left[\Phi\left(x^{\varepsilon}(T)\right)-\Phi(x(T))\right]+\mathbb{E}\left[\gamma\left(y^{\varepsilon}(0)\right)-\gamma(y(0))\right] .
\end{aligned}
$$

By (3.15), it is clear that $\mathbb{E}\left[\gamma\left(y^{\varepsilon}(0)\right)-\gamma\left(y(0)+y^{1}(0)\right)\right]=o(\varepsilon)$. Hence

$$
\mathbb{E}\left[\gamma\left(y^{\varepsilon}(0)\right)-\gamma(y(0))\right]=\mathbb{E}\left[\gamma\left(y(0)+y^{1}(0)\right)-\gamma(y(0))\right]+o(\varepsilon)=\mathbb{E}\left[\gamma_{y}^{\top}(y(0)) y^{1}(0)\right]+o(\varepsilon) .
$$

Similarly by (3.14), we have $\mathbb{E}\left[\Phi\left(x^{\varepsilon}(T)\right)-\Phi(x(T))\right]=\mathbb{E}\left[\Phi_{x}^{\top}(x(T)) x^{1}(T)\right]+o(\varepsilon)$. And by Lemma 3.4, we get

$$
\begin{aligned}
\mathbb{E} \int_{0}^{T} & {\left[l\left(t, x^{\varepsilon}(t), y^{\varepsilon}(t), z^{\varepsilon}(t), x^{\varepsilon}(t-\delta), u^{\varepsilon}(t), u^{\varepsilon}(t-\delta)\right)\right.} \\
& \left.-l\left(t, x(t)+x^{1}(t), y(t)+y^{1}(t), z(t)+z^{1}(t), x(t-\delta)+x^{1}(t-\delta), u^{\varepsilon}(t), u^{\varepsilon}(t-\delta)\right)\right] \mathrm{d} t=o(\varepsilon) .
\end{aligned}
$$

So by Lemma 3.3, using the technique to deal with the time delay term again, we obtain that

$$
\begin{aligned}
& \mathbb{E} \int_{0}^{T}\left[l\left(t, x^{\varepsilon}(t), y^{\varepsilon}(t), z^{\varepsilon}(t), x^{\varepsilon}(t-\delta), u^{\varepsilon}(t), u^{\varepsilon}(t-\delta)\right)-l(u)\right] \mathrm{d} t \\
= & \int_{0}^{T}\left[l\left(t, x(t)+x^{1}(t), y(t)+y^{1}(t), z(t)+z^{1}(t), x(t-\delta)+x^{1}(t-\delta), u^{\varepsilon}(t), u^{\varepsilon}(t-\delta)\right)-l(u)\right] \mathrm{d} t+o(\varepsilon) \\
= & \mathbb{E} \int_{0}^{T}\left[l_{x}^{\top} x^{1}(t)+l_{y}^{\top} y^{1}(t)+l_{z}^{\top} x^{1}(t)+l_{x(\delta)}^{\top} x^{1}(t-\delta)\right] \mathrm{d} t+\mathbb{E} \int_{0}^{T}\left[l\left(u^{\varepsilon}\right)-l(u)+\left(l_{x}\left(u^{\varepsilon}\right)-l_{x}(u)\right)^{\top} x^{1}(t)\right. \\
& \left.+\left(l_{y}\left(u^{\varepsilon}\right)-l_{y}(u)\right)^{\top} y^{1}(t)+\left(l_{z}\left(u^{\varepsilon}\right)-l_{z}(u)\right)^{\top} z^{1}(t)+\left(l_{x(\delta)}\left(u^{\varepsilon}\right)-l_{x(\delta)}(u)\right)^{\top} x^{1}(t-\delta)\right] \mathrm{d} t+o(\varepsilon) \\
= & \mathbb{E} \int_{0}^{T}\left[l_{x}^{\top} x^{1}(t)+l_{y}^{\top} y^{1}(t)+l_{z}^{\top} x^{1}(t)+l_{x(\delta)}^{\top} x^{1}(t-\delta)+l\left(u^{\varepsilon}\right)-l(u)\right] \mathrm{d} t+o(\varepsilon) .
\end{aligned}
$$

Sum the above three results up, it is easily to see that (3.20) holds. The proof is complete. 


\subsection{Proof of Theorem 2.1}

Proof of Theorem 2.1. Applying Itô's formula to $\left\langle x^{1}(\cdot), q(\cdot)\right\rangle+\left\langle y^{1}(\cdot), p(\cdot)\right\rangle$. By the adjoint equation (2.1), variational equation (3.1) and variational inequality (3.20), we get

$$
\begin{aligned}
& \mathbb{E} \int_{0}^{T}\left[l_{x}^{\top} x^{1}(t)+l_{y}^{\top} y^{1}(t)+l_{z}^{\top} z^{1}(t)+l_{x(\delta)}^{\top} x^{1}(t-\delta)+l\left(u^{\varepsilon}\right)-l(u)\right] \mathrm{d} t+\mathbb{E}\left[\Phi_{x}^{\top}(x(T)) x^{1}(T)\right]+\mathbb{E}\left[\gamma_{y}^{\top}(y(0)) y^{1}(0)\right] \\
= & \mathbb{E} \int_{0}^{T}\left[\left\langle q(t), b\left(u^{\varepsilon}\right)-b(u)\right\rangle-\left\langle p(t), f\left(u^{\varepsilon}\right)-f(u)\right\rangle+l\left(u^{\varepsilon}\right)-l(u)\right] \mathrm{d} t \\
= & \mathbb{E} \int_{0}^{T}\left[H\left(t, x(t), y(t), z(t), x(t-\delta), \mathbb{E}^{\mathcal{F}_{t}}[y(t+\delta)], u^{\varepsilon}(t), u^{\varepsilon}(t-\delta), p(t), q(t), k(t)\right)\right. \\
& \left.\quad-H\left(t, x(t), y(t), z(t), x(t-\delta), \mathbb{E}^{\mathcal{F}_{t}}[y(t+\delta)], u(t), u(t-\delta), p(t), q(t), k(t)\right)\right] \mathrm{d} t \\
:= & \mathbb{E} \int_{0}^{T}\left[H\left(t, u^{\varepsilon}(t), u^{\varepsilon}(t-\delta)\right)-H(t, u(t), u(t-\delta))\right] \mathrm{d} t \geq o(\varepsilon) .
\end{aligned}
$$

In the above, we have used the duality between SDDE and ABSDE such as the following:

$$
\begin{aligned}
& \mathbb{E} \int_{0}^{T}\left\langle y^{1}(t-\delta), f_{y(+\delta)}^{\top} p(t)\right\rangle \mathrm{d} t-\mathbb{E} \int_{0}^{T}\left\langle y^{1}(t), \mathbb{E}^{\mathcal{F}_{t}}\left(\left.f_{y(+\delta)}\right|_{t+\delta}\right)^{\top} p(t+\delta)\right\rangle \mathrm{d} t \\
= & \mathbb{E} \int_{-\delta}^{T-\delta}\left\langle y^{1}(t), \mathbb{E}^{\mathcal{F}_{t}}\left(\left.f_{y(+\delta)}\right|_{t+\delta}\right)^{\top} p(t+\delta)\right\rangle \mathrm{d} t-\mathbb{E} \int_{0}^{T}\left\langle y^{1}(t), \mathbb{E}^{\mathcal{F}_{t}}\left(\left.f_{y+(\delta)}\right|_{t+\delta}\right)^{\top} p(t+\delta)\right\rangle \mathrm{d} t \\
= & \mathbb{E} \int_{-\delta}^{0}\left\langle y^{1}(t), \mathbb{E}^{\mathcal{F}_{t}}\left(\left.f_{y(+\delta)}\right|_{t+\delta}\right)^{\top} p(t+\delta)\right\rangle \mathrm{d} t-\mathbb{E} \int_{T-\delta}^{T}\left\langle y^{1}(t), \mathbb{E}^{\mathcal{F}_{t}}\left(\left.f_{y(+\delta)}\right|_{t+\delta}\right)^{\top} p(t+\delta)\right\rangle \mathrm{d} t=0,
\end{aligned}
$$

since $p(t)=0$ for any $t \in[-\delta, 0)$ and $y^{1}(t)=0$ for any $t \in(T, T+\delta]$;

(ii)

$$
\begin{aligned}
& \mathbb{E} \int_{0}^{T}\left\langle x^{1}(t-\delta), b_{x(\delta)}^{\top} q(t)\right\rangle \mathrm{d} t-\mathbb{E} \int_{0}^{T}\left\langle x^{1}(t), \mathbb{E}^{\mathcal{F}_{t}}\left(b_{x(\delta)} \mid t+\delta\right)^{\top} q(t+\delta)\right\rangle \mathrm{d} t \\
= & \mathbb{E} \int_{-\delta}^{T-\delta}\left\langle x^{1}(t), \mathbb{E}^{\mathcal{F}_{t}}\left(\left.b_{x(\delta)}\right|_{t+\delta}\right)^{\top} q(t+\delta)\right\rangle \mathrm{d} t-\mathbb{E} \int_{0}^{T}\left\langle x^{1}(t), \mathbb{E}^{\mathcal{F}_{t}}\left(\left.b_{x(\delta)}\right|_{t+\delta}\right)^{\top} q(t+\delta)\right\rangle \mathrm{d} t \\
= & \mathbb{E} \int_{-\delta}^{0}\left\langle x^{1}(t), \mathbb{E}^{\mathcal{F}_{t}}\left(\left.b_{x(\delta)}\right|_{t+\delta}\right)^{\top} q(t+\delta)\right\rangle \mathrm{d} t-\mathbb{E} \int_{T-\delta}^{T}\left\langle x^{1}(t), \mathbb{E}^{\mathcal{F}_{t}}\left(\left.b_{x(\delta)}\right|_{t+\delta}\right)^{\top} q(t+\delta)\right\rangle \mathrm{d} t \\
= & 0
\end{aligned}
$$

since $x^{1}(t)=0$ for any $t \in[-\delta, 0]$ and $q(t)=0$ for any $t \in(T, T+\delta]$ and

$$
\begin{aligned}
& \mathbb{E} \int_{0}^{T}\left\langle x^{1}(t-\delta), \sigma_{x(\delta)}^{\top} k(t)\right\rangle \mathrm{d} t-\mathbb{E} \int_{0}^{T}\left\langle x^{1}(t), \mathbb{E}^{\mathcal{F}_{t}}\left(\left.\sigma_{x(\delta)}\right|_{t+\delta}\right)^{\top} k(t+\delta)\right\rangle \mathrm{d} t \\
= & \mathbb{E} \int_{-\delta}^{T-\delta}\left\langle x^{1}(t), \mathbb{E}^{\mathcal{F}_{t}}\left(\left.\sigma_{x(\delta)}\right|_{t+\delta}\right)^{\top} k(t+\delta)\right\rangle \mathrm{d} t-\mathbb{E} \int_{0}^{T}\left\langle x^{1}(t), \mathbb{E}^{\mathcal{F}_{t}}\left(\left.\sigma_{x(\delta)}\right|_{t+\delta}\right)^{\top} k(t+\delta)\right\rangle \mathrm{d} t \\
= & \mathbb{E} \int_{-\delta}^{0}\left\langle x^{1}(t), \mathbb{E}^{\mathcal{F}_{t}}\left(\left.\sigma_{x(\delta)}\right|_{t+\delta}\right)^{\top} k(t+\delta)\right\rangle \mathrm{d} t-\mathbb{E} \int_{T-\delta}^{T}\left\langle x^{1}(t), \mathbb{E}^{\mathcal{F}_{t}}\left(\left.\sigma_{x(\delta)}\right|_{t+\delta}\right)^{\top} k(t+\delta)\right\rangle \mathrm{d} t \\
= & 0
\end{aligned}
$$


since $x^{1}(t)=0$ for any $t \in[-\delta, 0]$ and $k(t)=0$ for any $t \in[T, T+\delta]$. Moreover, the following relation is also needed:

$$
\begin{aligned}
& \mathbb{E} \int_{0}^{T}\left\langle x^{1}(t-\delta), l_{x(\delta)}\right\rangle \mathrm{d} t-\mathbb{E} \int_{0}^{T}\left\langle x^{1}(t), \mathbb{E}^{\mathcal{F}_{t}}\left(\left.l_{x(\delta)}\right|_{t+\delta}\right)\right\rangle \mathrm{d} t \\
= & \mathbb{E} \int_{-\delta}^{T-\delta}\left\langle x^{1}(t), \mathbb{E}^{\mathcal{F}_{t}}\left(\left.l_{x(\delta)}\right|_{t+\delta}\right)\right\rangle \mathrm{d} t-\mathbb{E} \int_{0}^{T}\left\langle x^{1}(t), \mathbb{E}^{\mathcal{F}_{t}}\left(\left.l_{x(\delta)}\right|_{t+\delta}\right)\right\rangle \mathrm{d} t \\
= & \mathbb{E} \int_{-\delta}^{0}\left\langle x^{1}(t), \mathbb{E}^{\mathcal{F}_{t}}\left(\left.l_{x(\delta)}\right|_{t+\delta}\right)\right\rangle \mathrm{d} t-\mathbb{E} \int_{T-\delta}^{T}\left\langle x^{1}(t), \mathbb{E}^{\mathcal{F}_{t}}\left(\left.l_{x(\delta)}\right|_{t+\delta}\right)\right\rangle \mathrm{d} t \\
= & 0
\end{aligned}
$$

since $x^{1}(t)=0$ for any $t \in[-\delta, 0]$ and $l_{x(\delta)}(t)=0$ for any $t \in(T, T+\delta]$. Thus (2.4) can be obtained easily from (3.21) similar to [4]. We omit the detail. The proof is complete.

\subsection{Proof of Theorem 2.2}

In this subsection, we will prove Theorem 2.2. As mentioned in the introduction, our method is applying the technique of Clarke generalized gradient. The Clarke generalized gradient was used to derive sufficient conditions for stochastic optimal control problems by Zhou [30], Yong and Zhou [27]. For the readers' convenience, we firstly review some preliminaries of the Clarke generalized gradient which will play a key role in this section.

Let $v: \mathcal{X} \rightarrow R$ be a locally Lipschitz continuous function, where $\mathcal{X}$ is a convex set in $\mathbf{R}^{n}$. We recall the following definition.

Definition 3.6 ([30]). The Clarke generalized gradient of $v$ at $\hat{x} \in \mathcal{X}$, denoted by $\partial v(\hat{x})$, is a set defined by

$$
\partial v(\hat{x}):=\left\{\zeta \in \mathbf{R}^{n} ;\langle\zeta, \xi\rangle \leq v^{0}(\hat{x} ; \xi) \text {, for any } \xi \in \mathbf{R}^{n}\right\},
$$

where

$$
v^{0}(\hat{x} ; \xi):=\limsup _{x \in \mathcal{X}, x+h \xi \in \mathcal{X}, x \rightarrow \hat{x}, h \rightarrow 0+} \frac{h(x+h \xi)-h(x)}{h} .
$$

The following results are useful in this section.

Lemma 3.7 ([30]). The following properties hold:

(1) $\partial v(\hat{x})$ is a nonempty convex set and satisfying $\partial(-v)(\hat{x})=-\partial v(\hat{x})$;

(2) for any set $N \subset \mathcal{X}$ of measure zero

$$
\partial v(\hat{x})=c o\left\{\lim _{i \rightarrow \infty} v_{x}\left(x_{i}\right): v \text { is differentiable at } x_{i}, x_{i} \notin N \text { and } x_{i} \rightarrow \hat{x}\right\},
$$

where "co" denotes the convex hull of a set;

(3) if $\hat{x}$ attains maximum or minimum of $v$ over $\mathcal{X}$, then $0 \in \partial v(\hat{x})$;

(4) if $v$ is a convex (respectively, concave) function, then $p \in \partial v(\hat{x})$ if and only if

$$
v(x)-v(\hat{x}) \geq(\text { respectively, } \leq)\langle p, x-\hat{x}\rangle,
$$

for any $x \in \mathcal{X}$.

Lemma 3.8 ([30]). Let $\rho$ be a convex or concave function on $\mathbf{R}^{d} \times \mathbf{U}$. Assume that $\rho(x, u)$ is Lipschitz continuous in $u$, differentiable in $x$, and $\rho_{x}(x, u)$ is continuous in $(x, u)$. For a given $(\bar{x}, \bar{u}) \in \mathbf{R}^{d} \times \mathbf{U}$, if $r \in \partial_{u} \rho(\bar{x}, \bar{u})$, then $\left(\rho_{x}(\bar{x}, \bar{u}), r\right) \in \partial_{x, u} \rho(\bar{x}, \bar{u})$. 
Proof of Theorem 2.2.. Let $u(\cdot)$ be an admissible control and $(x(\cdot), y(\cdot), z(\cdot))$ be the corresponding trajectory. Applying Itô's formula to $\left\langle q(t), x^{v}(t)-x(t)\right\rangle+\left\langle p(t), y^{v}(t)-y(t)\right\rangle$, noting control system (1.1), adjoint equation (2.1) and Hamiltonian function (2.2), we get

$$
\begin{aligned}
& \mathbb{E}\left[\Phi_{x}(x(T))^{\top}\left(x^{v}(T)-x(T)\right)\right]+\mathbb{E}\left[\gamma_{y}(y(0))^{\top}\left(y^{v}(0)-y(0)\right)\right] \\
= & \mathbb{E} \int_{0}^{T}\left[\left\langle q(t), b\left(t, x^{v}(t), y^{v}(t), z^{v}(t), x^{v}(t-\delta), v(t), v(t-\delta)\right)-b(u)\right\rangle\right. \\
& -\left\langle p(t), f\left(t, x^{v}(t), y^{v}(t), z^{v}(t), \mathbb{E}^{\mathcal{F}_{t}}\left[y^{v}(t+\delta)\right], v(t), v(t-\delta)\right)-f(u)\right\rangle \\
& \left.+\operatorname{tr}\left\{k(t)^{\top}\left(\sigma\left(t, x^{v}(t), y^{v}(t), z^{v}(t), x^{v}(t-\delta)\right)-\sigma(t, x(t), y(t), z(t), x(t-\delta))\right)\right\}\right] \mathrm{d} t \\
& +\mathbb{E} \int_{0}^{T}\left\{\left\langle x^{v}(t)-x(t),-H_{x}(t)-\mathbb{E}^{\mathcal{F}_{t}}\left[\left.H_{x(\delta)}(t)\right|_{t+\delta}\right]\right\rangle\right. \\
& \left.-\left\langle y^{v}(t)-y(t), H_{y}(t)+H_{y_{(+\delta)}}(t-\delta)\right\rangle-\left\langle z^{v}(t)-z(t), H_{z}(t)\right\rangle\right\} \mathrm{d} t .
\end{aligned}
$$

By the convexity of $\Phi$ and $\gamma$, we derive that

$$
\begin{aligned}
J(v(\cdot))-J(u(\cdot))= & \mathbb{E} \int_{0}^{T}\left[l\left(t, x^{v}(t), y^{v}(t), z^{v}(t), x^{v}(t-\delta), v(t), v(t-\delta)\right)-l(u)\right] \mathrm{d} t \\
& +\mathbb{E}\left[\Phi\left(x^{v}(T)\right)-\Phi(x(T))\right]+\mathbb{E}\left[\gamma\left(y^{v}(0)\right)-\gamma(y(0))\right] \\
\geq & \mathbb{E}\left[\Phi_{x}(x(T))^{\top}\left(x^{v}(T)-x(T)\right)\right]+\mathbb{E}\left[\gamma_{y}(y(0))^{\top}\left(y^{v}(0)-y(0)\right)\right] \\
& +\mathbb{E} \int_{0}^{T}\left[H\left(t, x^{v}(t), y^{v}(t), z^{v}(t), \mathbb{E}^{\mathcal{F}_{t}}\left[y^{v}(t+\delta)\right], v(t-\delta), p(t), q(t), k(t)\right)-H(t)\right] \mathrm{d} t \\
& +\mathbb{E} \int_{0}^{T}\left\{\left\langle x^{v}(t)-x(t),-H_{x}(t)-\mathbb{E}^{\mathcal{F}_{t}}\left[\left.H_{x(\delta)}(t)\right|_{t+\delta}\right]\right\rangle\right. \\
& \left.-\left\langle y^{v}(t)-y(t), H_{y}(t)+H_{y(+\delta)}(t-\delta)\right\rangle-\left\langle z^{v}(t)-z(t), H_{z}(t)\right\rangle\right\} \mathrm{d} t .
\end{aligned}
$$

Denote by $\partial_{(x, y, z, x(\delta), y(+\delta), v, v(\delta))} H(t)$, etc., the Clarke generalized gradients of $H$ evaluated at $(x(t), y(t), z(t)$, $\left.x(t-\delta), \mathbb{E}^{\mathcal{F}_{t}}[y(t+\delta)], v(t), v(t-\delta)\right)$. Maximum condition $(2.4)$ yields $(0,0) \in \partial_{(v, v(\delta))} H(t)$, a.e., a.s. By Lemma 3.7, $\left(\partial_{(x, y, z, x(\delta), y(+\delta))} H(t), 0,0\right) \in \partial_{(x, y, z, x(\delta), y(+\delta), v, v(\delta))} H(t)$, a.e., a.s. By Lemma 3.6 (4) and the convexity of $H$ in $(x, y, z, x(\delta), y(+\delta))$, it follows that

$$
\begin{aligned}
& H\left(t, x^{v}(t), y^{v}(t), z^{v}(t), x^{v}(t-\delta), \mathbb{E}^{\mathcal{F}_{t}}\left[y^{v}(t+\delta)\right], v(t), v(t-\delta), p(t), q(t), k(t)\right)-H(t) \\
\geq & \left\langle x^{v}(t)-x(t), H_{x}(t)\right\rangle+\left\langle x^{v}(t-\delta)-x(t-\delta), H_{x(\delta)}(t)\right\rangle+\left\langle y^{v}(t)-y(t), H_{y}(t)\right\rangle \\
& +\left\langle\mathbb{E}^{\mathcal{F}_{t}}\left[y^{v}(t+\delta)-y(t+\delta)\right], H_{y(+\delta)}(t)\right\rangle .
\end{aligned}
$$

Noticing that

$$
\begin{aligned}
& \mathbb{E} \int_{0}^{T}\left\langle x^{v}(t)-x(t), \mathbb{E}^{\mathcal{F}_{t}}\left[\left.H_{x(\delta)}(t)\right|_{t+\delta}\right]\right\rangle \mathrm{d} t-\mathbb{E} \int_{0}^{T}\left\langle x^{v}(t-\delta)-x(t-\delta), H_{x_{\delta}}(t)\right\rangle \mathrm{d} t \\
= & \mathbb{E} \int_{0}^{T}\left\langle x^{v}(t)-x(t), \mathbb{E}^{\mathcal{F}_{t}}\left[\left.H_{x(\delta)}(t)\right|_{t+\delta}\right]\right\rangle \mathrm{d} t-\mathbb{E} \int_{-\delta}^{T-\delta}\left\langle x^{v}(t)-x(t), \mathbb{E}^{\mathcal{F}_{t}}\left[\left.H_{x(\delta)}(t)\right|_{t+\delta}\right]\right\rangle \mathrm{d} t \\
= & \mathbb{E} \int_{-\delta}^{0}\left\langle x^{v}(t)-x(t), \mathbb{E}^{\mathcal{F}_{t}}\left[\left.H_{x(\delta)}(t)\right|_{t+\delta}\right]\right\rangle \mathrm{d} t+\mathbb{E} \int_{T-\delta}^{T}\left\langle x^{v}(t)-x(t), \mathbb{E}^{\mathcal{F}_{t}}\left[\left.H_{x(\delta)}(t)\right|_{t+\delta}\right]\right\rangle \mathrm{d} t=0,
\end{aligned}
$$


since $x^{v}(t)=x(t)=\xi(t)$ for any $t \in[-\delta, 0]$ and $H_{x(\delta)}(t)=0$ for any $t \in(T, T+\delta]$. And similarly, noticing that

$$
\begin{aligned}
& \mathbb{E} \int_{0}^{T}\left\langle y^{v}(t)-y(t), H_{y(+\delta)}(t-\delta)\right\rangle \mathrm{d} t-\mathbb{E} \int_{0}^{T}\left\langle\mathbb{E}^{\mathcal{F}_{t}}\left[y^{v}(t+\delta)-y(t+\delta)\right], H_{y(+\delta)}(t)\right\rangle \mathrm{d} t \\
= & \mathbb{E} \int_{-\delta}^{T-\delta}\left\langle\mathbb{E}^{\mathcal{F}_{t}}\left[y^{v}(t+\delta)-y(t+\delta)\right], H_{y(+\delta)}(t)\right\rangle \mathrm{d} t-\mathbb{E} \int_{0}^{T}\left\langle\mathbb{E}^{\mathcal{F}_{t}}\left[y^{v}(t+\delta)-y(t+\delta)\right], H_{y(+\delta)}(t)\right\rangle \mathrm{d} t \\
= & \mathbb{E} \int_{-\delta}^{0}\left\langle\mathbb{E}^{\mathcal{F}_{t}}\left[y^{v}(t+\delta)-y(t+\delta)\right], H_{y(+\delta)}(t)\right\rangle \mathrm{d} t-\mathbb{E} \int_{T-\delta}^{T}\left\langle\mathbb{E}^{\mathcal{F}_{t}}\left[y^{v}(t+\delta)-y(t+\delta)\right], H_{y(+\delta)}(t)\right\rangle \mathrm{d} t=0,
\end{aligned}
$$

since $y^{v}(t)=y(t)=\varphi(t)$ for any $t \in(T, T+\delta]$ and $H_{y(+\delta)}(t)=0$ for any $t \in[-\delta, 0)$. Then we have $J(v(\cdot))-$ $J(u(\cdot)) \geq 0, \forall v(\cdot) \in \mathcal{U}_{a d}$. Thus $u(\cdot)$ is the optimal control. The proof is complete.

\section{EXAMPleS: LINEAR-QUADRATIC CASE}

In this section, we give two LQ examples to illustrate our results in Section 3. The first example is discussed in Section 4.1 in which the control system coefficients contain only delayed and anticipated state terms. In Section 4.2 we deal with the case when the control system coefficients involve both delayed and anticipated state terms and control terms with delay. In both cases, the explicit optimal controls are obtained.

\subsection{Systems with delayed and anticipated states}

Let us consider the following linear stochastic control system with delayed and anticipated states $(n=m=1)$ :

$$
\left\{\begin{array}{l}
\mathrm{d} x^{v}(t)=\left[A(t) x^{v}(t)-b y^{v}(t)+D(t-\delta) x^{v}(t-\delta)+C(t) v(t)\right] \mathrm{d} t+E(t) \mathrm{d} W(t), t \in[0, T], \\
-\mathrm{d} y^{v}(t)=\left[a x^{v}(t)+A(t) y^{v}(t)+D(t) \mathbb{E}^{\mathcal{F}_{t}}\left[y^{v}(t+\delta)\right]+F(t) v(t)\right] \mathrm{d} t-z^{v}(t) \mathrm{d} W(t), t \in[0, T], \\
x^{v}(t)=\xi(t), t \in[-\delta, 0], \quad y^{v}(T)=c x^{v}(T), y^{v}(t)=\varphi(t), t \in(T, T+\delta] .
\end{array}\right.
$$

For any given $v(\cdot)$, it is easy to show that the $G$-monotonic condition (H2) holds. Then AFBSDDE (4.1) admits a unique solution $\left(x^{v}(\cdot), y^{v}(\cdot), z^{v}(\cdot)\right)$. The cost functional is

$$
J(v(\cdot))=\frac{1}{2} \mathbb{E}\left\{\int_{0}^{T}\left[R(t) x^{v}(t)^{2}+\tilde{R}(t) y^{v}(t)^{2}+L(t) v^{2}(t)\right] \mathrm{d} t+M_{T} x^{v}(T)^{2}+N_{0} y^{v}(0)^{2}\right\} .
$$

Our optimal control problem is to minimize (4.2) over $v(\cdot) \in \mathcal{U}_{a d}$ subject to (4.1). In the above, constants $a>0, b \geq 0, c>0, M_{T} \geq 0, N_{0} \geq 0$. Functions $L(\cdot)>0, A(\cdot), C(\cdot), E(\cdot), F(\cdot), R(\cdot), \tilde{R}(\cdot)$ defining on $[0, T]$ are bounded and deterministic, $L^{-1}(\cdot)$ is also bounded. Function $D(t), t \in[-\delta, T+\delta]$ satisfies $D(t)=D, t \in$ $[-\delta, 0], D(t)=0, t \in[T, T+\delta]$. Deterministic functions $\xi(\cdot), \varphi(\cdot)$ satisfy $\int_{-\delta}^{0} \xi(t)^{2} \mathrm{~d} t<+\infty, \int_{T}^{T+\delta} \varphi(t)^{2} \mathrm{~d} t<+\infty$. $W(\cdot)$ is a one-dimensional standard Brownian motion on $\left(\Omega, \mathcal{F},\left\{\mathcal{F}_{t}\right\}, \mathbf{P}\right)$. Admissible control set denoted by $\mathcal{U}_{a d}$ is the set of all $\mathcal{F}_{t}$-adapted square integrable process $v(t), t \in[0, T]$ valued in $\mathbf{U} \subset \mathbf{R}$.

Now, the Hamiltonian function (2.2) is given by

$$
\begin{aligned}
& H(t, x, y, z, x(\delta), y(+\delta), v, p, q, k)=q[A(t) x-b y+D(t-\delta) x(\delta)+C(t) v] \\
& \quad-p[a x+A(t) y+D(t) y(+\delta)+F(t) v]+k E(t)+\frac{1}{2} R(t) x^{2}+\frac{1}{2} \tilde{R}(t) y^{2}+\frac{1}{2} L(t) v^{2} .
\end{aligned}
$$

According to Theorem 2.1, if $u(\cdot)$ is optimal and $(x(\cdot), y(\cdot), z(\cdot))$ is the optimal trajectory, then

$$
u(t)=-L^{-1}(t)[C(t) q(t)-F(t) p(t)], t \in[0, T],
$$


where $(p(\cdot), q(\cdot))$ is the solution of the following adjoint equation:

$$
\left\{\begin{array}{l}
\mathrm{d} p(t)=[A(t) p(t)+b q(t)-D(t-\delta) p(t-\delta)-\tilde{R}(t) y(t)] \mathrm{d} t, t \in[0, T], \\
-\mathrm{d} q(t)=\left[-a p(t)+A(t) q(t)+D(t) \mathbb{E}^{\mathcal{F}_{t}}[q(t+\delta)]+R(t) x(t)\right] \mathrm{d} t-k(t) \mathrm{d} W(t), t \in[0, T], \\
p(0)=-N_{0} y(0), p(t)=0, \quad t \in[-\delta, 0), \\
q(T)=-c p(T)+M_{T} x(T), k(T)=0, \quad q(t)=0, k(t)=0, t \in(T, T+\delta] .
\end{array}\right.
$$

However, it is difficult to verify this AFBSDDE (4.4) admits a unique solution $(p(\cdot), q(\cdot), k(\cdot))$. Because the coefficients and terminal condition of (4.4) are coupled with $(x(\cdot), y(\cdot), z(\cdot))$ which now is the solution of the following

$$
\left\{\begin{aligned}
\mathrm{d} x(t)=\left[A(t) x(t)-b y(t)+D(t-\delta) x(t-\delta)-C(t) L(t)^{-1} C(t) q(t)\right. \\
\left.+C(t) L(t)^{-1} F(t) p(t)\right] \mathrm{d} t+E(t) \mathrm{d} W(t), t \in[0, T] \\
-\mathrm{d} y(t)=\left[a x(t)+A(t) y(t)+D(t) \mathbb{E}^{\mathcal{F}_{t}}[y(t+\delta)]-F(t) L(t)^{-1} C(t) q(t)\right. \\
\left.\quad+F(t) L(t)^{-1} F(t) p(t)\right] \mathrm{d} t-z(t) \mathrm{d} W(t), t \in[0, T], \\
x^{v}(t)=\xi(t), t \in[-\delta, 0], \quad y^{v}(T)=c x^{v}(T), y^{v}(t)=\varphi(t), t \in(T, T+\delta] .
\end{aligned}\right.
$$

Noting that AFBSDDE (4.5) is coupled with $(p(\cdot), q(\cdot), k(\cdot))$ vice versa! However, we can rewrite the above two coupled AFBSDDEs (4.4) and (4.5) together:

$$
\left\{\begin{aligned}
& \mathrm{d} p(t)=[A(t) p(t)+b q(t)-D(t-\delta) p(t-\delta)-\tilde{R}(t) y(t)] \mathrm{d} t, t \in[0, T], \\
& \mathrm{d} x(t)=\left[A(t) x(t)-b y(t)+D(t-\delta) x(t-\delta)-C(t) L(t)^{-1} C(t) q(t)\right. \\
&\left.+C(t) L(t)^{-1} F(t) p(t)\right] \mathrm{d} t+E(t) \mathrm{d} W(t), t \in[0, T], \\
&-\mathrm{d} q(t)= {\left[-a p(t)+A(t) q(t)+D(t) \mathbb{E}^{\mathcal{F}_{t}}[q(t+\delta)]+R(t) x(t)\right] \mathrm{d} t-k(t) \mathrm{d} W(t), t \in[0, T], } \\
&-\mathrm{d} y(t)= {\left[a x(t)+A(t) y(t)+D(t) \mathbb{E}^{\mathcal{F}_{t}}[y(t+\delta)]-F(t) L(t)^{-1} C(t) q(t)\right.} \\
&\left.\quad+F(t) L(t)^{-1} F(t) p(t)\right] \mathrm{d} t-z(t) \mathrm{d} W(t), t \in[0, T], \\
& p(0)=- N_{0} y(0), q(T)=-c p(T)+M_{T} x(T), y(T)=c x(T), k(T)=0, \\
& x(t)= \xi(t), p(t)=0, t \in[-\delta, 0) ; q(t)=0, k(t)=0, y(t)=\varphi(t), t \in(T, T+\delta] .
\end{aligned}\right.
$$

This is an AFBSDDE with double dimensions. See Yu [28] for general theory of this kind equations without delay. We declare that if AFBSDDE (4.6) admits a unique solution $(x(\cdot), y(\cdot), z(\cdot), p(\cdot), q(\cdot), k(\cdot))$ then we can check that all the conditions in Theorem 2.2 are satisfied. Consequently, control (4.3) is really optimal.

Moreover, we can prove that any admissible control $u(\cdot)$ of the form $(4.3)$ is optimal by the completion of squares technique. In fact, for any admissible control $v(\cdot)$, applying Itô's formula to $\left(x^{v}(t)-x(t)\right) q(t)+\left(y^{v}(t)-\right.$ $y(t)) p(t)$, noting that (4.1), (4.4) and using the duality relation to deal with delay term as before, we can get

$$
\mathbb{E}\left[M_{T} x(T)\left(x^{v}(T)-x(T)\right)\right]+\mathbb{E}\left[N_{0} y(0)\left(y^{v}(0)-y(0)\right)\right]=\mathbb{E} \int_{0}^{T}[C(t) q(t)-M(t) p(t)](v(t)-u(t)) \mathrm{d} t .
$$

Then, as $L(t)>0, \forall t \in[0, T], M_{T} \geq 0, N_{0} \geq 0$, we have

$$
\begin{aligned}
J(v(\cdot))-J(u(\cdot))= & \frac{1}{2} \mathbb{E} \int_{0}^{T}\left[R(t)\left(x^{v}(t)-x(t)\right)^{2}+2 R(t) x(t)\left(x^{v}(t)-x(t)\right)+\tilde{R}(t)\left(y^{v}(t)-y(t)\right)^{2}\right. \\
& \left.+2 \tilde{R}(t) y(t)\left(y^{v}(t)-y(t)\right)+L(t)(v(t)-u(t))^{2}+2 L(t) u(t)(v(t)-u(t))\right] \mathrm{d} t \\
& +\mathbb{E}\left\{M_{T}\left(x^{v}(T)-x(T)\right)^{2}+2 M_{T} x(T)\left(x^{v}(T)-x(T)\right)+N_{0}\left(y^{v}(0)-y(0)\right)^{2}+2 N_{0} y(0)\left(y^{v}(0)-y(0)\right)\right\} \\
\geq & \mathbb{E}\left\{\int_{0}^{T} L(t) u(t)(v(t)-u(t)) \mathrm{d} t+M_{T} x(T)\left(x^{v}(T)-x(T)\right)+N_{0} y(0)\left(y^{v}(0)-y(0)\right)\right\} \\
= & \mathbb{E} \int_{0}^{T}[L(t) u(t)+C(t) q(t)-M(t) p(t)](v(t)-u(t)) \mathrm{d} t=0 .
\end{aligned}
$$


Since fully coupled AFBSDDE with double dimensions (4.6) is complicated, we continue to discuss it for some special case. We choose $R(\cdot)=\tilde{R}(\cdot)=M_{T} \equiv 0$ in $(4.2)$ :

$$
J(v(\cdot))=\frac{1}{2} \mathbb{E}\left\{\int_{0}^{T} L(t) v^{2}(t) \mathrm{d} t+N_{0} y^{v}(0)^{2}\right\} .
$$

The adjoint equation (4.4) reduces to

$$
\left\{\begin{array}{l}
\mathrm{d} p(t)=[A(t) p(t)+b q(t)+D(t-\delta) p(t-\delta)] \mathrm{d} t, t \in[0, T], \\
-\mathrm{d} q(t)=\left[-a p(t)+A(t) q(t)+\mathbb{E}^{\mathcal{F}_{t}}[D(t+\delta) q(t+\delta)]\right] \mathrm{d} t-k(t) \mathrm{d} W(t), t \in[0, T], \\
p(0)=-N_{0} y(0), p(t)=0, \quad t \in[-\delta, 0), \\
q(T)=-c p(T), k(T)=0, \quad q(t)=0, k(t)=0, t \in(T, T+\delta] .
\end{array}\right.
$$

Fortunately, it is easy to verify that the $G$-monotonic condition (H2)' holds. Then (4.8) admits a unique solution $(p(\cdot), q(\cdot), k(\cdot))$. Consequently, $u(t)=-L^{-1}(t)(C(t) q(t)-F(t) p(t))$ is really optimal by the same approach, and our problem can be solved completely. To summarize, we have the following result.

Theorem 4.1. Let $R(\cdot)=\tilde{R}(\cdot)=M_{T} \equiv 0$. Then (4.3) is the unique optimal control for our LQ problem (4.1), (4.2), where $(p(\cdot), q(\cdot), k(\cdot))$ is the unique solution of the adjoint AFBSDDE (4.8).

\subsection{Systems involving both delayed/anticipated states and delayed controls}

Furthermore in this subsection, let us consider the following linear stochastic control system involving both delayed/anticipated states and delay controls $(n=m=1)$ :

$$
\left\{\begin{array}{c}
\mathrm{d} x^{v}(t)=\left[A(t) x^{v}(t)-b y^{v}(t)+D(t-\delta) x^{v}(t-\delta)\right. \\
+C(t) v(t)+\tilde{C}(t) v(t-\delta)] \mathrm{d} t+E(t) \mathrm{d} W(t), t \in[0, T] \\
-\mathrm{d} y^{v}(t)=\left[a x^{v}(t)+A(t) y^{v}(t)+D(t) \mathbb{E}^{\mathcal{F}_{t}}\left[y^{v}(t+\delta)\right]\right. \\
\quad+F(t) v(t)+\tilde{F}(t) v(t-\delta)] \mathrm{d} t-z^{v}(t) \mathrm{d} W(t), t \in[0, T], \\
x^{v}(t)=\xi(t), t \in[-\delta, 0], \quad y^{v}(T)=c x^{v}(T), y^{v}(t)=\varphi(t), t \in(T, T+\delta] .
\end{array}\right.
$$

Let $\mathcal{U}_{a d}$ denote the set of admissible controls $v(\cdot)$ of the form:

$$
v(t)=\left\{\begin{array}{l}
\eta(t), t \in[-\delta, 0] \\
v(t) \in L_{\mathcal{F}}^{2}([0, T] ; \mathbf{R}) \text { and } v(t) \in \mathbf{R}, t \in[0, T], \text { a.s. }
\end{array}\right.
$$

Our optimal control problem is to minimize the following quadratic cost functional

$$
J(v(\cdot))=\frac{1}{2} \mathbb{E}\left\{\int_{0}^{T}\left[R(t) x^{v}(t)^{2}+\tilde{R}(t) y^{v}(t)^{2}+L(t) v^{2}(t)\right] \mathrm{d} t+M_{T} x^{v}(T)^{2}+N_{0} y^{v}(0)^{2}\right\}
$$

over $v(\cdot) \in \mathcal{U}_{a d}$ subject to $(4.9)$. In the above, functions $\tilde{C}(\cdot), \tilde{M}(\cdot)$ defining on $[0, T]$ are bounded and deterministic. Other parameters are the same as in Section 4.1.

For any given $v(\cdot)$, similarly we can verify that the $G$-monotonic condition (H2) holds. Then AFBSDDE (4.9) admits a unique solution $\left(x^{v}(\cdot), y^{v}(\cdot), z^{v}(\cdot)\right)$ and the Hamiltonian function (2.2) is given by

$$
\begin{aligned}
& H(t, x, y, z, x(\delta), y(+\delta), v, v(\delta), p, q, k)=q[A(t) x-b y+D(t-\delta) x(\delta)+C(t) v+\tilde{C}(t) v(\delta)] \\
& \quad-p[a x+A(t) y+D(t) y(+\delta)+F(t) v+\tilde{F}(t) v(\delta)]+k E(t)+\frac{1}{2} R(t) x^{2}+\frac{1}{2} \tilde{R}(t) y^{2}+\frac{1}{2} L(t) v^{2} .
\end{aligned}
$$


According to Theorem 2.1, if $u(\cdot)$ is optimal and $(x(\cdot), y(\cdot), z(\cdot))$ is the optimal trajectory, then

$$
u(t)=-L^{-1}(t)\left\{C(t) q(t)-F(t) p(t)+\mathbb{E}^{\mathcal{F}_{t}}[\tilde{C}(t+\delta) q(t+\delta)-\tilde{F}(t+\delta) p(t+\delta)]\right\}, t \in[0, T],
$$

where $(p(\cdot), q(\cdot))$ is the solution of the same adjoint equation (4.4). Moreover, it is now coupled with $(x(\cdot), y(\cdot)$, $z(\cdot))$ which is the solution of the following AFBSDDE

$$
\left\{\begin{aligned}
\mathrm{d} x(t)= & \left\{A(t) x(t)-b y(t)+D(t-\delta) x(t-\delta)-C(t) L(t)^{-1}[C(t) q(t)+F(t) p(t)\right. \\
+ & \left.\mathbb{E}^{\mathcal{F}_{t}}[\tilde{C}(t+\delta) q(t+\delta)-\tilde{F}(t+\delta) p(t+\delta)]\right]-\tilde{C}(t) L(t-\delta)^{-1} \\
& \times[C(t-\delta) q(t-\delta)+F(t-\delta) p(t-\delta)+\tilde{C}(t) q(t)-\tilde{F}(t) p(t)]\} \mathrm{d} t+E(t) \mathrm{d} W(t), t \in[\delta, T], \\
\mathrm{d} x(t)= & \left\{A(t) x(t)-b y(t)+D(t-\delta) x(t-\delta)-C(t) L(t)^{-1}[C(t) q(t)+F(t) p(t)\right. \\
+ & \left.\left.\mathbb{E}^{\mathcal{F}_{t}}[\tilde{C}(t+\delta) q(t+\delta)-\tilde{F}(t+\delta) p(t+\delta)]\right]+\tilde{C}(t) \eta(t-\delta)\right\} \mathrm{d} t+E(t) \mathrm{d} W(t), t \in[0, \delta], \\
-\mathrm{d} y(t)= & \left\{a x(t)+A(t) y(t)+D(t) \mathbb{E}^{\mathcal{F}_{t}}[y(t+\delta)]-F(t) L(t)^{-1}[C(t) q(t)+F(t) p(t)\right. \\
& \left.+\mathbb{E}^{\mathcal{F}_{t}}[\tilde{C}(t+\delta) q(t+\delta)-\tilde{F}(t+\delta) p(t+\delta)]\right]-\tilde{F}(t) L(t-\delta)^{-1} \\
& \times[C(t-\delta) q(t-\delta)+F(t-\delta) p(t-\delta)+\tilde{C}(t) q(t)-\tilde{F}(t) p(t)]\} \mathrm{d} t-z(t) \mathrm{d} W(t), t \in[\delta, T], \\
-\mathrm{d} y(t)= & \left\{a x(t)+A(t) y(t)+D(t) \mathbb{E}^{\mathcal{F}_{t}}[y(t+\delta)]-F(t) L(t)^{-1}[C(t) q(t)+F(t) p(t)\right. \\
& \left.\left.+\mathbb{E}^{\mathcal{F}_{t}}[\tilde{C}(t+\delta) q(t+\delta)-\tilde{F}(t+\delta) p(t+\delta)]\right]+\tilde{F}(t) \eta(t-\delta)\right\} \mathrm{d} t-z(t) \mathrm{d} W(t), t \in[0, \delta], \\
x^{v}(t)= & \xi(t), v(t)=\eta(t), t \in[-\delta, 0], \\
y^{v}(T)= & c x^{v}(T), y^{v}(t)=\varphi(t), t \in(T, T+\delta] .
\end{aligned}\right.
$$

Similarly AFBSDDE (4.12) is coupled with $(p(\cdot), q(\cdot), k(\cdot))$ vice versa! And if we rewrite (4.4) and (4.12) together, then we encounter another more complicated AFBSDDE with double dimensions as in Section 4.1. Moreover, by Theorem 2.2 we know that control variable (4.11) is really optimal. On the other hand, we can prove any admissible control $u(\cdot)$ of the form (4.11) is optimal directly by the completion of squares technique.

Finally, we consider the cost functional (4.7) with $R(\cdot)=\tilde{R}(\cdot)=M_{T} \equiv 0$. The adjoint AFBSDDE (4.4) reduces to (4.8) which is unique solvable. Then (4.11) is really optimal and our problem can be solved completely. To summarize, we have the following result.

Theorem 4.2. Let $R(\cdot)=\tilde{R}(\cdot)=M_{T} \equiv 0$. Then (4.11) is the unique optimal control for our LQ problem (4.9), (4.10), where $(p(\cdot), q(\cdot), k(\cdot))$ is the unique solution of the adjoint AFBSDDE (4.8).

\section{CONCLUding REMARKS}

In this paper, we have discussed the optimal control problem in which the control system is described by a fully coupled AFBSDDE. The maximum principle is obtained under the assumption that the diffusion coefficient does not contain the control variables and the control domain is not necessarily convex. Both the necessary and sufficient conditions of optimality are proved. As illustrating examples, two kinds of linear quadratic control problems are discussed and both optimal controls are derived explicitly.

As one referee pointed out, our results depend heavily on the $G$-monotonic assumption (H2). In fact, in the past several years, the research on the wellposedness of FBSDEs have made rapid progress. For example, Zhang [29] impose some kinds of simple weak-coupled conditions to obtain the existence and uniqueness for fully 
coupled FBSDEs. For AFBSDDEs, results on this direction are few and the only existing result is by Chen and Wu [6] using the $G$-monotonic assumption (H2). We wish to study optimal control problems for fully coupled AFBSDDEs (including FBSDEs) without the $G$-monotonic assumption in the future.

An interesting and challenging open problem is to extend the results in this paper to the case in which the diffusion coefficient contains both control variable and its delayed term. Noting that this bring rather difficulty to obtain the corresponding maximum principle even without delay. Preferable progress has been made by Yong [26] recently for one kind general coupled forward-backward stochastic control systems with mixed initial-terminal conditions without delay. We will study this topic for delayed system in our future research.

Acknowledgements. The authors would like to thank the anonymous referees for many constructive comments that led to three improved versions of the paper. The authors also thank the associate editor and the corresponding editor for their efficient handling of this paper. Especial thanks are devoted to Prof. Zhen Wu, Dr. Zhiyong Yu and Dr. Li Chen for many useful discussions. Finally, the second author thanks Department of Applied Mathematics, The Hong Kong Polytechnic University, for their hospitality during his visits to Hong Kong.

\section{REFERENCES}

[1] F. Antonelli, Backward-forward stochastic differential equations. Ann. Appl. Prob. 3 (1993) 777-793.

[2] F. Antonelli, E. Baruccib and M.E. Mancinoc, Asset pricing with a forward-backward stochastic differential utility. Econ. Lett. 72 (2001) 151-157.

[3] R. Buckdahn and Y. Hu, Hedging contingent claims for a large investor in an incomplete market. Adv. Appl. Prob. 30 (1998) 239-255.

[4] L. Chen and Z. Wu, Maximum principle for stochastic optimal control problem of forward-backward system with delay, in Proc. Joint 48th IEEE CDC and 28th CCC, Shanghai, P.R. China (2009) 2899-2904.

[5] L. Chen and Z. Wu, Maximum principle for the stochastic optimal control problem with delay and application. Automatica 46 (2010) 1074-1080.

[6] L. Chen and Z. Wu, A type of generalized forward-backward stochastic differential equations and applications. Chin. Ann. Math. Ser. B 32 (2011) 279-292.

[7] D. Cucoo and J. Cvitanic, Optimal consumption choices for a 'large' investor. J. Econ. Dyn. Control 22 (1998) $401-436$.

[8] J. Cvitanic and J. Ma, Hedging options for a large investor and forward-backward SDE's. Ann. Appl. Prob. 6 (1996) $370-398$.

[9] J. Cvitanic, X.H. Wan and J.F. Zhang, Optimal contracts in continuous-time models. J. Appl. Math. Stoch. Anal. 2006 (2006), Article ID 95203 1-27.

[10] N. El Karoui, S.G. Peng and M.C. Quenez, Backward stochastic differential equations in finance. Math. Finance 7 (1997) $1-71$.

[11] Y. Hu and S.G. Peng, Solution of forward-backward stochastic differential equations. Prob. Theory Relat. Fields 103 (1995) $273-283$

[12] V.B. Kolmanovsky and T.L. Maizenberg, Optimal control of stochastic systems with aftereffect, in Stochastic Systems, Translated from Avtomatika $i$ Telemekhanika. 1 (1973) 47-61.

[13] Q.X. Meng, A maximum principle for optimal control problem of fully coupled forward-backward stochastic systems with partial information. Sciences in China, Mathematics 52 (2009) 1579-1588.

[14] S.E.A. Mohammed, Stochastic differential equations with memory: theory, examples and applications. Stochastic Analysis and Related Topics VI. The Geido Workshop, 1996. Progress in Probability, Birkhauser (1998).

[15] B. Øksendal and A. Sulem, A maximum principle for optimal control of stochastic systems with delay, with applications to finance, Optimal Control and Partial Differential Equations - Innovations and Applications, edited by J.M. Menaldi, E. Rofman and A. Sulem. IOS Press, Amsterdam (2000).

[16] E. Pardoux and S.G. Peng, Adapted solution of a backward stochastic differential equation. Syst. Control Lett. 14 (1990) $55-61$.

[17] S.G. Peng, Backward stochastic differential equations and applications to optimal control. Appl. Math. Optim. 27 (1993) $125-144$.

[18] S.G. Peng, Backward stochastic differential equations-stochastic optimization theory and viscosity solutions of HJB equations, Topics on Stochastic Analysis (in Chinese), edited by J.A. Yan, S.G. Peng, S.Z. Fang and L.M. Wu. Science Press, Beijing (1997) 85-138.

[19] S.G. Peng and Z. Wu, Fully coupled forward-backward stochastic differential equations and applications to the optimal control. SIAM J. Control Optim. 37 (1999) 825-843.

[20] S.G. Peng and Z. Yang, Anticipated backward stochastic differential equations. Ann. Prob. 37 (2009) $877-902$.

[21] J.T. Shi and Z. Wu, The maximum principle for fully coupled forward-backward stochastic control systems. ACTA Automatica Sinica 32 (2006) 161-169. 
[22] J.T. Shi and Z. Wu, The maximum principle for partially observed optimal control of fully coupled forward-backward stochastic system. J. Optim. Theory Appl. 145 (2010) 543-578.

[23] G.C. Wang and Z. Wu, The maximum principles for stochastic recursive optimal control problems under partial information. IEEE Trans. Autom. Control 54 (2009) 1230-1242.

[24] N. Williams, On dynamic principal-agent problems in continuous time. Working paper (2008). Available on the website: http:// www.ssc.wisc.edu/ ${ }^{n}$ nilliam/dynamic-pa1.pdf

[25] Z. Wu, Maximum principle for optimal control problem of fully coupled forward-backward stochastic systems. Syst. Sci. Math. Sci. 11 (1998) 249-259.

[26] J.M. Yong, Optimality variational principle for controlled forward-backward stochastic differential equations with mixed initialterminal conditions. SIAM J. Control Optim. 48 (2010) 4119-4156.

[27] J.M. Yong and X.Y. Zhou, Stochastic Controls: Hamiltonian Systems and HJB Equations. Springer-Verlag, New York (1999).

[28] Z.Y. Yu, Linear-quadratic optimal control and nonzero-sum differential game of forward-backward stochastic system. Asian J. Control 14 (2012) 1-13.

[29] J.F. Zhang, The wellposedness of FBSDEs. Discrete Contin. Dyn. Syst., Ser. B 6 (2006) 927-940.

[30] X.Y. Zhou, Sufficient conditions of optimality for stochastic systems with controllable diffusions. IEEE Trans. Autom. Control 41 (1996) 1176-1179. 